\title{
Effects of Static Magnetic Field Exposure on Blood Flow
}

\author{
Dima Abi Abdallah ${ }^{1}$, Agnès Drochon ${ }^{1}$, Vincent Robin $^{2}$, and Odette Fokapu ${ }^{13}$ \\ 1 Laboratoire de Biomécanique et Bioingénierie, Université de Technologie de Compiègne, France \\ 2 Laboratoire de Mathématiques Appliquées, Université de Technologie de Compiègne, France \\ 3 Université de Picardie Jules-Verne, IUT de L'Aisne, France
}

\begin{abstract}
This paper investigates the influence of static magnetic field exposure on blood flow. We mainly focus on steady flows in a rigid vessel and review the existing theoretical solutions, each based on some simplifying hypothesis. The results are developed, examined and compared, showing how the magnetohydrodynamic interactions reduce the flow rate and generate electric voltages across the vessel walls. These effects are found to be moderate for magnetic fields such as those used in magnetic resonance imaging. In this case, a very simplified solution, formulated by neglecting the walls conductivity as well as the induced magnetic fields, is proven suitable.
\end{abstract}

PACS. 41.20.-q Electromagnetism - 47.63.-b Biological fluid dynamics - 47.65.-d Magnetohydrodynamics and electrohydrodynamics

\section{Introduction}

Studying the effects of magnetic fields on human physiology has raised great interests over the past years, especially now that the wide medical use of Magnetic Resonance Imaging (MRI) [1] is constantly increasing the exposures to strong static fields. Besides some subtle biological effects upon cell division at very high field [2], documented experiments have shown that the manifested biological effects of a static magnetic field are mostly due to blood Correspondence to: dima.abiabdallah@utc.fr flow in the large arteries. When the body is subjected to a magnetic field, the positively and negatively charged blood particles, flowing transversally to the field, get deflected by the Lorentz force in opposite directions. This induces electrical currents and voltages across the vessel walls and in the surrounding tissues, strong enough to be detected at the surface of the thorax in the electrocardiogram (ECG) [3]. In the cardiac MRI gating context, where the $\mathrm{R}$ peaks of the simultaneously recorded ECG are used to trigger the image acquisition sequences, these 
voltages can be particularly hindering for synchronization as they might lead to incorrect peak detections $[4,5]$. Furthermore, the interactions between the induced currents and the applied magnetic field can cause a reduction of flow rate and, hence, a reactive compensatory increase in blood pressure in order to retain a constant volume flow rate $[6]$. Magnetic field interactions with blood flow have been demon-model for blood flow in vessels. In this case, the most strated by multiple authors throughout in vitro experiments as well as in vivo studies. Keltner et al.[7] and Tasu et al. [8] measured pressure and flow rate using in vitro experimental setups. Gaffey et al. [9] and Tenforde et al. [10] observed animal ECG alterations, manifested mainly as $\mathrm{T}$ wave elevations. Weikl et al. [11] measured changes due to superimposed voltages on animal and human ECG. More recently, Abi Abdallah et al. [12] characterized temporal and spectral alterations of human ECG, measuring $\mathrm{T}$ wave amplitudes, and showing a signal energy increase and a spectral shift toward low frequencies. Jehenson et al. [13] observed variations of heart frequency at $2 \mathrm{~T}$ and indicated that the sinus node might be affected. Subsequent studies [11], [14] reported no such changes of heart rates in static fields up to 8T. Chakeres et al. [14] assessed the effects of magnetic fields on human vital signs and essentially noted an arterial pressure increase. Holden [15] in his study of the heart sensitivity to induced potentials predicted notable effects on pacemaker rate for static fields greater than $8 \mathrm{~T}$.

Theoretical magnetohydrodynamic blood flow calculations have, however, been addressed much earlier and go back as far as the early sixties. Korchevskii et al.[16] proposed a velocity profile solution for blood flow between two parallel plates under a constant pressure gradient with a perpendicular magnetic field, under the assumption that blood is newtonian. Other studies focused on flow in a rigid circular tube with non conducting walls placed in a transverse magnetic field, in order to offer a more realistic complete solution of the magnetohydrodynamic equations of a conducting fluid was proposed by Gold [17]. Setting a constant pressure gradient, Gold derived expressions for the velocity profile as well as for the induced magnetic field. Vardanyan [6] subsequently published an approximate steady solution where the velocity profile and flow rate were calculated by neglecting the induced fields. More recent studies have been essentially based on these founding works, such as the study of Keltner et al. [7], where a comparison was established between the results of Gold and Vardanyan to assess the consequences of neglecting the inductions. With the same hypothesis as Vardanyan, Sud et al. [18] later dealt with a sinusoidal pressure gradient to describe the pulsed nature of blood flow in arteries, and recently, Abi Abdallah et al. [19] proposed a more realistic arterial flow solution using a physiological pressure gradient model. Both solutions, however, assumed the arteries to be rigid vessels. In a follow-up to their first paper, Sud et al. [20] took into account the compliant nature of large arteries and studied the flow in an elastic tube where they calculated expressions for the velocity profiles and pressure waves. The hypothesis of conducting walls was 
only introduced by Kinouchi et al. [21] who included inductions in the vessel and the surrounding tissues, in the steady flow case, to evaluate the ECG superimposed voltages, using finite elements.

In this paper we review the steady magnetohydrodynamics solutions in a rigid vessel. We analyze the exact solution presented by Gold for which we show new illustrations and interpretations, and then develop it further to show the separation of charges across the vessel walls. Afterward, we consider the approximate solution of Vardanyan and complete it with induced potential calculations. These solutions are then compared to each other and to the results of Kinouchi et al., obtained for conducting walls, in order to discuss the influence of the simplifying assumptions on calculations.

\section{General equations}

The flow of a newtonian, incompressible, conducting fluid in an externally applied magnetic field is governed by the laws of magnetohydrodynamics. It is defined by a coupling of Maxwell's quasi-static electromagnetic equations and Ohm's law, on the one hand,

$$
\begin{gathered}
\nabla \cdot \mathbf{E}=\frac{\rho_{e}}{\epsilon}, \\
\nabla \wedge \mathbf{E}=-\frac{\partial \mathbf{B}}{\partial t}, \\
\nabla \cdot \mathbf{B}=0, \\
\nabla \wedge \mathbf{B}=\mu \mathbf{j}, \\
\mathbf{j}=\sigma(\mathbf{E}+\mathbf{u} \wedge \mathbf{B}),
\end{gathered}
$$

and the Navier-Stokes and conservation equations on the other,

$$
\begin{gathered}
\rho\left(\frac{\partial \mathbf{u}}{\partial t}+(\mathbf{u} \cdot \nabla) \mathbf{u}\right)=-\nabla p+\eta \Delta \mathbf{u}+\mathbf{j} \wedge \mathbf{B} \\
\nabla \cdot \mathbf{u}=0
\end{gathered}
$$

Where $\mathbf{u}$ and $p$ represent the fluid velocity $(\mathrm{m} / \mathrm{s})$ and pressure $(P a), \mathbf{B}$ and $\mathbf{E}$ are the magnetic $(T)$ and electric $(\mathrm{V} / \mathrm{m})$ fields, and $\mathbf{j}$ is the electric current density $\left(\mathrm{A} / \mathrm{m}^{2}\right)$. With $\epsilon$ the electric permittivity $(F / m), \rho_{e}$ the charge density $\left(C / m^{3}\right), \mu$ the magnetic permeability $(H / m)$ and $\eta$, $\sigma, \rho$ respectively the fluid viscosity $(P a . s)$, conductivity $(S / m)$ and density $\left(\mathrm{kg} / \mathrm{m}^{3}\right)$.

By substituting $\mathbf{j}$ with its expression given in (4), the Navier-Stokes equation yields,

$$
\rho\left(\frac{\partial \mathbf{u}}{\partial t}+(\mathbf{u} \cdot \nabla) \mathbf{u}\right)=-\nabla p+\eta \Delta \mathbf{u}+\frac{1}{\mu}(\nabla \wedge \mathbf{B}) \wedge \mathbf{B}
$$

Moreover, by calculating the curl of (5), then using (2) and (4), we can establish the induction equation,

$$
\frac{\partial \mathbf{B}}{\partial t}=\nabla \wedge(\mathbf{u} \wedge \mathbf{B})-\frac{1}{\sigma \mu} \nabla \wedge(\nabla \wedge \mathbf{B}),
$$

which can also be written as,

$$
\frac{\partial \mathbf{B}}{\partial t}=\nabla \wedge(\mathbf{u} \wedge \mathbf{B})+\frac{1}{\sigma \mu} \Delta \mathbf{B}
$$

using the formula $\mathbf{a} \wedge(\mathbf{b} \wedge \mathbf{c})=(\mathbf{a} \cdot \mathbf{c}) \mathbf{b}-(\mathbf{a} \cdot \mathbf{b}) \mathbf{c}$, with $\nabla \cdot \mathbf{B}=0$

Thus, characterizing the magnetohydrodynamic flow of a newtonian, incompressible, conducting fluid sums up to the resolution of the following system of equations,

$$
\left\{\begin{array}{l}
\rho\left(\frac{\partial \mathbf{u}}{\partial t}+(\mathbf{u} \cdot \nabla) \mathbf{u}\right)=-\nabla p+\eta \Delta \mathbf{u}+\frac{1}{\mu}(\nabla \wedge \mathbf{B}) \wedge \mathbf{B}, \\
\frac{\partial \mathbf{B}}{\partial t}=\nabla \wedge(\mathbf{u} \wedge \mathbf{B})+\frac{1}{\sigma \mu} \Delta \mathbf{B} .
\end{array}\right.
$$


Assuming that the blood is newtonian, in what follows we consider the case of a unidirectional flow of blood in a rigid circular vessel, under the influence of a transverse magnetic field (figure 1). The velocity and magnetic field are expressed as, $\mathbf{u}=(0,0, u(t, r, \theta))$ and $\mathbf{B}=\left(B_{0} \cos \theta,-B_{0} \sin \theta, B_{I}(t, r, \theta)\right)$, with $B_{I}<<B_{0}$. The induced magnetic field is parallel to the flow and ensures $\nabla \cdot \mathbf{B}=0$. In fact, the charge separation is expected to occur in a plane that is perpendicular to the velocity, thus producing an electric field oriented in that same plane. Therefore, $\nabla \wedge \mathbf{E}$ which is equal to $-\frac{\partial \mathbf{B}}{\partial t}$ would be parallel to $O z$.

The projection of (10) on $\mathbf{e}_{z}$, written in cylindrical coordinates, gives,

$$
\left\{\begin{array}{l}
\rho \frac{\partial u}{\partial t}=-\frac{\partial p}{\partial z}+\eta \Delta u+\frac{B_{0}}{\mu}\left(\cos \theta \frac{\partial B_{I}}{\partial r}-\frac{\sin \theta}{r} \frac{\partial B_{I}}{\partial \theta}\right), \\
\frac{\partial B_{I}}{\partial t}=B_{0}\left(\cos \theta \frac{\partial u}{\partial r}-\frac{\sin \theta}{r} \frac{\partial u}{\partial \theta}\right)+\frac{1}{\sigma \mu} \Delta B_{I},
\end{array}\right.
$$

supposing that the pressure gradient is only $z$-dependent.

This equation system might be expressed in a non-dimensional form by introducing the Hartmann, the Reynolds, and the magnetic Reynolds numbers,

$$
H_{a}=B_{0} a \sqrt{\frac{\sigma}{\eta}} \quad, \quad R_{e}=\frac{\rho u_{0} a}{\eta} \quad, \quad R_{m}=a u_{0} \sigma \mu \quad,
$$

and performing substitutions such as,

$$
\tilde{U}=\frac{u}{u_{0}}, \tilde{r}=\frac{r}{a}, \tilde{B}=\frac{B_{I}}{B_{0}}, \tau=t \frac{u_{0}}{a}, G=-\frac{a^{2}}{\eta u_{0}} \frac{\partial p}{\partial z},
$$

where $a$ represents the vessel radius, and $u_{0}$ is some characteristic mean velocity.

Equations (11) thus become,

$$
\left\{\begin{array}{l}
R_{e} \frac{\partial \tilde{U}}{\partial \tau}=G+\Delta \tilde{U}+\frac{H_{a}^{2}}{R_{m}}\left[\cos \theta \frac{\partial \tilde{B}}{\partial \tilde{r}}-\frac{\sin \theta}{\tilde{r}} \frac{\partial \tilde{B}}{\partial \theta}\right] \\
R_{m} \frac{\partial \tilde{B}}{\partial \tau}=\Delta \tilde{B}+R_{m}\left[\cos \theta \frac{\partial \tilde{U}}{\partial \tilde{r}}-\frac{\sin \theta}{\tilde{r}} \frac{\partial \tilde{U}}{\partial \theta}\right] .
\end{array}\right.
$$

For a steady magnetohydrodynamic flow, the non-dimensional system can be formulated [17] as,

$$
\left\{\begin{array}{l}
\Delta \tilde{U}+\frac{H_{a}^{2}}{R_{m}}\left[\cos \theta \frac{\partial \tilde{B}}{\partial \tilde{r}}-\frac{\sin \theta}{\tilde{r}} \frac{\partial \tilde{B}}{\partial \theta}\right]=-G, \\
\Delta \tilde{B}+R_{m}\left[\cos \theta \frac{\partial \tilde{U}}{\partial \tilde{r}}-\frac{\sin \theta}{\tilde{r}} \frac{\partial \tilde{U}}{\partial \theta}\right]=0 .
\end{array}\right.
$$

\section{Exact solution}

Gold [17] established an exact solution for (13) while considering non conducting vessel walls. This hypothesis constitutes a good approximation since, as stated by [7], the vascular tissues are about 6 times more resistive than blood. After decoupling the two equations, Gold resolved the problem using Fourier series, with boundary conditions such as $\tilde{U}(1, \theta)=0$ and $\tilde{B}(1, \theta)=0$, and formulated the following expressions for the velocity and induced magnetic field,

$$
\begin{aligned}
\tilde{U}(\tilde{r}, \theta)= & \frac{G}{2 H_{a}}\left[e^{-\frac{H_{a}}{2} \tilde{r} \cos \theta} \sum_{n=0}^{\infty} \epsilon_{n} \frac{I_{n}^{\prime}\left(\frac{H_{a}}{2}\right)}{I_{n}\left(\frac{H_{a}}{2}\right)} I_{n}\left(\frac{H_{a}}{2} \tilde{r}\right) \cos n \theta\right. \\
& \left.+e^{\frac{H_{a}}{2} \tilde{r} \cos \theta} \sum_{n=0}^{\infty}(-1)^{n} \epsilon_{n} \frac{I_{n}^{\prime}\left(\frac{H_{a}}{2}\right)}{I_{n}\left(\frac{H_{a}}{2}\right)} I_{n}\left(\frac{H_{a}}{2} \tilde{r}\right) \cos n \theta\right],
\end{aligned}
$$

$$
\begin{aligned}
\tilde{B}(\tilde{r}, \theta)= & \frac{R_{m} G}{2 H_{a}^{2}}\left[e^{-\frac{H_{a}}{2} \tilde{r} \cos \theta} \sum_{n=0}^{\infty} \epsilon_{n} \frac{I_{n}^{\prime}\left(\frac{H_{a}}{2}\right)}{I_{n}\left(\frac{H_{a}}{2}\right)} I_{n}\left(\frac{H_{a}}{2} \tilde{r}\right) \cos n \theta\right. \\
& -e^{\frac{H_{a}}{2} \tilde{r} \cos \theta} \sum_{n=0}^{\infty}(-1)^{n} \epsilon_{n} \frac{I_{n}^{\prime}\left(\frac{H_{a}}{2}\right)}{I_{n}\left(\frac{H_{a}}{2}\right)} I_{n}\left(\frac{H_{a}}{2} \tilde{r}\right) \cos n \theta \\
& -2 \tilde{r} \cos \theta]
\end{aligned}
$$

where $I_{n}$ is the $n^{\text {th }}$ order modified Bessel function of the first kind, and

$$
\epsilon_{n}=\left\{\begin{array}{l}
1 \text { for } n=0, \\
2 \text { for } n>0 .
\end{array}\right.
$$

Note that expanding (14) for small values of $H_{a}$ gives, $\tilde{U}(\tilde{r})=\frac{G}{4}\left(1-\tilde{r}^{2}\right)$, when $H_{a} \rightarrow 0$. Reverting to dimensional values we get, $u_{z}(r)=-\frac{\partial p / \partial z}{4 \eta}\left(a^{2}-r^{2}\right)$, which is 
none other than the classical Poiseuille flow profile, obtained in the absence of magnetic fields.

The non-dimensional mean velocity, defined as the ratio of the flow rate, $q=\iint_{A} u d S$ to the cross section, $A=\pi a^{2}$, is given by,

$$
\overline{\tilde{U}}=\frac{1}{\pi} \int_{0}^{2 \pi} \int_{0}^{1} \tilde{U} \tilde{r} d \tilde{r} d \theta .
$$

Integrating (14) yields,

$$
\begin{aligned}
\overline{\tilde{U}}= & G \sum_{n=0}^{\infty}(-1)^{n} \epsilon_{n} \frac{I_{n}^{\prime}\left(\frac{H_{a}}{2}\right)}{H_{a} I_{n}\left(\frac{H_{a}}{2}\right)}\left[\left(1+\frac{n^{2}}{\left(\frac{H_{a}}{2}\right)^{2}}\right) I_{n}^{2}\left(\frac{H_{a}}{2}\right)\right. \\
& \left.-I_{n}^{\prime 2}\left(\frac{H_{a}}{2}\right)\right] .
\end{aligned}
$$

Using the velocity and magnetic fields formulas, we can then draw up expressions for the induced current, electric field and voltage.

Equation (4) gives,

$$
\mathbf{j}(\tilde{r}, \theta)=\frac{1}{\mu} \nabla \wedge \mathbf{B}=\frac{1}{\mu} \frac{B_{0}}{a}\left(\frac{1}{\tilde{r}} \frac{\partial \tilde{B}}{\partial \theta} \mathbf{e}_{\mathbf{r}}-\frac{\partial \tilde{B}}{\partial \tilde{r}} \mathbf{e}_{\theta}\right) .
$$

Defining $j_{0}=\frac{1}{\mu} \frac{B_{0}}{a}$, we write in a non-dimensional form,

$$
\tilde{\mathbf{j}}(\tilde{r}, \theta)=\frac{\mathbf{j}(\tilde{r}, \theta)}{j_{0}}=\frac{1}{\tilde{r}} \frac{\partial \tilde{B}}{\partial \theta} \mathbf{e}_{\mathbf{r}}-\frac{\partial \tilde{B}}{\partial \tilde{r}} \mathbf{e}_{\theta} .
$$

Likewise, the electric field can be retrieved from (5),

$$
\begin{aligned}
\mathbf{E}(\tilde{r}, \theta) & =\frac{\mathbf{j}}{\sigma}-\mathbf{u} \wedge \mathbf{B} \\
& =B_{0} u_{0}\left[\left(\frac{1}{\tilde{r} R_{m}} \frac{\partial \tilde{B}}{\partial \theta}-\tilde{U} \sin \theta\right) \mathbf{e}_{\mathbf{r}}\right. \\
& \left.-\left(\frac{1}{R_{m}} \frac{\partial \tilde{B}}{\partial \tilde{r}}+\tilde{U} \cos \theta\right) \mathbf{e}_{\theta}\right] .
\end{aligned}
$$

With $E_{0}=B_{0} u_{0}$, we write,

$$
\begin{aligned}
\tilde{\mathbf{E}}(\tilde{r}, \theta)= & \left(\frac{1}{\tilde{r} R_{m}} \frac{\partial \tilde{B}}{\partial \theta}-\tilde{U} \sin \theta\right) \mathbf{e}_{\mathbf{r}} \\
& -\left(\frac{1}{R_{m}} \frac{\partial \tilde{B}}{\partial \tilde{r}}+\tilde{U} \cos \theta\right) \mathbf{e}_{\theta} .
\end{aligned}
$$

$$
\begin{aligned}
\rho_{e} & =-\epsilon \nabla \cdot(\mathbf{u} \wedge \mathbf{B}) \\
\Rightarrow \rho_{e}(\tilde{r}, \theta) & =-\epsilon \frac{B_{0} u_{0}}{a}\left(\sin \theta \frac{\partial \tilde{U}}{\partial \tilde{r}}+\frac{\cos \theta}{\tilde{r}} \frac{\partial \tilde{U}}{\partial \theta}\right) .
\end{aligned}
$$

Setting $\rho_{e_{0}}=\epsilon \frac{B_{0} u_{0}}{a}=\epsilon \frac{E_{0}}{a}$, we write the dimensionless charge density as,

$$
\tilde{\rho_{e}}(\tilde{r}, \theta)=-\left(\sin \theta \frac{\partial \tilde{U}}{\partial \tilde{r}}+\frac{\cos \theta}{\tilde{r}} \frac{\partial \tilde{U}}{\partial \theta}\right) .
$$

The electric potential, induced on the vessel walls, can be evaluated by integrating the radial component of the electric field for a given value of $\theta$. The highest voltage amplitude is attained for $\theta=\pi / 2$,

$$
V=2 \int_{0}^{a} E_{r}(r, \pi / 2) d r=2 u_{0} B_{0} a \int_{0}^{1} \tilde{E}_{r}(\tilde{r}, \pi / 2) d \tilde{r}
$$

With $V_{0}=2 u_{0} B_{0} a$, we get,

$$
\tilde{V}=\int_{0}^{1} \tilde{E}_{r}(\tilde{r}, \pi / 2) d \tilde{r}
$$

where,

$$
\left.\tilde{E}_{r}(\tilde{r}, \pi / 2)=\frac{1}{\tilde{r} R_{m}} \frac{\partial \tilde{B}}{\partial \theta}\right]_{\theta=\pi / 2}-\tilde{U}(\tilde{r}, \pi / 2) .
$$

Using the expressions of $\tilde{B}$ and $\tilde{U}$ given by (14) and (15), $\tilde{E}_{r}(\tilde{r}, \pi / 2)$ can be calculated as,

$$
\begin{aligned}
\tilde{E}_{r}(\tilde{r}, \pi / 2) & =-\frac{G}{H_{a}}\left[\frac{1}{2} \sum_{k=0}^{\infty}(-1)^{k} \epsilon_{k} \frac{I_{2 k}^{\prime}\left(\frac{H_{a}}{2}\right)}{I_{2 k}\left(\frac{H_{a}}{2}\right)} I_{2 k}\left(\frac{H_{a}}{2} \tilde{r}\right)-\frac{1}{H_{a}}\right. \\
& \left.+\frac{2}{\tilde{r} H_{a}} \sum_{k=0}^{\infty}(-1)^{k}(2 k+1) \frac{I_{2 k+1}^{\prime}\left(\frac{H_{a}}{2}\right)}{I_{2 k+1}\left(\frac{H_{a}}{2}\right)} I_{2 k+1}\left(\frac{H_{a}}{2} \tilde{r}\right)\right] .
\end{aligned}
$$

The results illustrated hereafter are computed in Matlab using the numerical parameters shown in Table 1 . In this case, the Hartmann number would relate to the static magnetic field intensity by $B_{0} \approx 9 H_{a}$. 


\subsection{Velocity profiles}

In this section we present some illustrations of velocity results. Figures 2 and 3 compare the 3D profiles and contours for various intensities of $B_{0}$. They show that the presence of an external magnetic field tends to reduce the blood flow and flatten the velocity profile. For typical MRI fields (up to $8 T$ ) the retardation and flattening effects are almost indiscernible (figures 2(b) and 2(c)). At $1.5 T$ the peak velocity is reduced by $0.09 \%$ only, and at $8 T$ the reduction is slighter than $2.5 \%$. Contrarily, for $B_{0}=20 T$, $30 T$ and $40 T$ a clear reduction of the velocity amplitude can be noted in figures 2(d), 2(e) and 2(f), where the profile is flattened while being stretched parallel to the direction of $B_{0}$ (figures 3(d), 3(e) and 3(f)). By comparing figures $2(\mathrm{a})$ and $2(\mathrm{f})$ we can observe a peak velocity reduction of $34.5 \%$. This agrees very well with the calculations of Keltner et al. [7] who noted a 30\% reduction for $H_{a}=4$.

Figure 4 shows the velocity profiles plotted versus $\tilde{r}$ for $\theta=0$, and $\theta=\pi / 2$, using various $H_{a}$ values. For $H_{a}=0$ we recognize the Poiseuille profile plots, which are identical in both planes. Then, as noted above, when $H_{a}$ increases, the velocity is reduced and the curve is flattened. For high field intensities the flattening is more significant in the plane $\theta=0$, i.e. parallel to $B_{0}$, whereas for small $H_{a}$ the axial symmetry is almost preserved. Figure 5 plots the decrease of the mean velocity as $H_{a}$ augments. For $H_{a}=4.47$, for example, we get a $25 \%$ reduction, while, as mentioned earlier, the peak velocity is cut down by $34.5 \%$. This can only confirm the flattening process.
Using conducting walls, Kinouchi et al. [21] evaluated a $4.93 \%$ mean velocity decrease for $H_{a}=1$, whereas here the mean velocity is reduced by only $2 \%$. This is almost equivalent to the authors' computed reduction when the walls conductivity is divided by 10 (a reduction inferior to $2.6 \%$ was reported). In fact, contrarily to the case of conducting walls where the current exits the vessel and is conducted to the neighboring tissues, the insulating walls would capture the induced currents and force them to circulate on the inner side of the vessel, generating current loops [21]. Currents circulating in the opposite direction to the charge separation, induce a compensating Lorentz force which leads to an underestimation of flow retardation. This underestimation gap widens as $H_{a}$ increases (figure 5).

Note that for a Poiseuille flow, the mean velocity is given by $\overline{\tilde{U}}_{\text {pois }}=\frac{a^{2}}{8 \eta u_{0}} \frac{\Delta P}{L}$, thus, $\frac{\overline{\tilde{U}}_{\text {pois }}}{G}=\frac{1}{8}$. This is the exact value traced in figure 5 when $H_{a}=0$.

\subsection{Induced magnetic fields}

Figures 6 and 7 show the induced magnetic fields intensities for three values of $B_{0}$. The plots present two lobes on each side of the main current line : one in the same direction as the flow, and another opposite to the flow (the charge separation happens along $\mathrm{Oy}$, perpendicular to both the flow and the static field). The shapes are somewhat identical for low and high intensities, but the lobe magnitude increases with the applied field. It remains however very weak, even for $B_{0}=40 T$ it does not ex- 
ceed $4 \times 10^{-7} T$ (lower than earth's magnetic field which amounts to $\left.5 \times 10^{-5} T\right)$.

Figure 8, matching Gold's figure 3, plots the non-dimensional induced field versus $\tilde{r}$ in the plane $\theta=0\left(B_{I}=0\right.$ in $\theta=\pi / 2$ ), for several values of $H_{a}$. The induced field increases as we move away from the center, to attain a maximum, then decreases to become null on the insulating walls. This is consistent with the lobe shape traced in figure 6 . It can be noticed that the induced field value is not proportional to the applied field. In fact, when $B_{0}$ increases, the flow is retarded further, therefore reducing the inductions. This can be confirmed by observing the evolution of the induced field as $B_{0}$ changes in figure 9 . For small applied intensities, the induction augments with $B_{0}$ then peaks around $B_{0}=50 T\left(H_{a} \approx 5.5\right)$. Beyond that, flow reductions become very considerable and the induced fields weaken.

\subsection{Induced current densities}

Figure 10 shows the $\tilde{j}_{r}$ and $\tilde{j}_{\theta}$ components of the induced current density. In the vessel center, $\tilde{j}_{r}$ is predominant along the $O y$ direction and $\tilde{j}_{\theta}$ is small, whereas in the vicinity of the walls $\tilde{j}_{\theta}$ is prominent and $\tilde{j}_{r}$ is null. This indicates that the current flows mainly in the center parallel to $O y$, once its comes near the walls it loops downward (figure 11), thus conforming to the hypothesis of non conducting walls: the current is unable to exit the vessel and circulates in a closed loop on the inner side of the walls [7]. As for the current amplitude, it is proportional to $B_{0}$, as shown in figure 12 . Using the parameters of Ki- nouchi et al. [21] $\left(\eta=5 \cdot 10^{-3}\right.$ Pa.s and $\left.u_{0}=0.6 \mathrm{~m} / \mathrm{s}\right)$, we find, for $B_{0}=1 T$, a current density of approximately $150 \mathrm{~mA} / \mathrm{m}^{2}$ near the center. This value is clearly inferior to the one computed by [21] who report current densities of approximately $200 \mathrm{~m} A / \mathrm{m}^{2}$. This difference is most likely due to the compensating current circulation opposite to the charge separation direction.

\subsection{Induced electric fields, charge densities and wall potentials}

The transverse components of the induced electric field shown in figure 13, indicate that, as would be expected, the field is primarily oriented along $-\mathbf{e}_{\mathbf{y}}$, in opposite direction to the main current (from the positive charges toward the negative ones). Its amplitude increases with $B_{0}$, it is the highest near the vessel center and weakens as we move away vertically toward the walls (figure 14). Hence the Lorentz force acts strongest at the center.

Figure 15, presenting the charge density, shows how the positive and negative particles are pushed apart by the Lorentz force, thus forming symmetric concentrations on either side of the $O x$ axis. The separation is better emphasized when $B_{0}$ augments : the concentrations intensify, and the charges are pushed further against the walls.

The induced voltage across the vessel walls for $\theta=\pi / 2$ is presented in figure 16. For small $H_{a}$ values, $V$ increases rapidly with $B_{0}$, whereas for very strong static magnetic fields the curve slope becomes a lot less steep. In fact, since the potential is proportional to both the magnetic 
field and the velocity, for high fields where the flow is significantly reduced, the augmentation of $B_{0}$ is somewhat compensated by the velocity reduction.

Note that, by using the parameters of [21], for $B_{0}=1 T$ we get a $12 m V$ voltage amplitude, while Kinouchi et al. give $4.4 m V$ on the aortic wall at $\theta=\pi / 2$, hence a potential difference across the wall of $8.8 \mathrm{mV}$. Our insulating walls assumption, has therefore led to a voltage overestimation of almost $36 \%$. For higher magnetic field values, a larger overestimation should be expected, given that the gap between the flow rates would be more substantial.

\section{Approximate solution}

\subsection{Velocity profiles}

Vardanyan [6] advanced that for low $R_{m}$ number flows, such as in the case of blood flow, an approximate solution of the problem might be formulated by neglecting the induced fields. In this case, the flow would only be defined by the Navier-Stokes equation where $\mathbf{j}$ is given by Ohm's law (5) with $\mathbf{E}<<\mathbf{u} \wedge \mathbf{B}_{0}$ (no external electric field). By replacing $\mathbf{j}=\sigma \mathbf{u} \wedge \mathbf{B}_{0}$ in equation (6), the projection on the $O z$ axis of the Navier-Stokes steady equation in cylindrical coordinates take on the form,

$$
\frac{\partial p}{\partial z}=\eta\left(\frac{1}{r} \frac{\partial u}{\partial r}+\frac{\partial^{2} u}{\partial r^{2}}\right)-\sigma u B_{0}^{2}
$$

Using non-dimensional parameters, we write,

$$
\Delta \tilde{U}-H_{a}^{2} \tilde{U}=-G
$$

with the boundary condition $\tilde{U}(1)=0$.

The solution of this differential equation can be formu- lated as,

$$
\tilde{U}=\frac{G}{H_{a}^{2}}\left(1-\frac{I_{0}\left(H_{a} \tilde{r}\right)}{I_{0}\left(H_{a}\right)}\right) .
$$

The mean velocity in this case would be,

$$
\overline{\tilde{U}}=2 \frac{G}{H_{a}^{2}}\left(\frac{1}{2}-\frac{I_{1}\left(H_{a}\right)}{H_{a} I_{0}\left(H_{a}\right)}\right) .
$$

Figure 17 compares the velocity profiles computed using the approximate solution (25), on the one hand, and Gold's exact solution (14) for $\theta=0$ and $\theta=\pi / 2$, on the other hand. One might notice that neglecting the induced fields overestimates the flow retardation, especially for strong fields, and disregards the $\theta$ asymmetrical nature of the profile. This overestimation can also be noted in figure 18 showing the mean velocity plots. When the induced fields are neglected, the mean velocity tends more rapidly to zero as $H_{a}$ increases. In fact, the approximate solution only deals with the consequences of a current circulating perpendicular to the flow and to the external magnetic field: $\mathbf{j}=\sigma \mathbf{u} \wedge \mathbf{B}$, which defines the charge separation. Contrarily, the exact solution, also includes the current recirculation by using $\mathbf{j}=\frac{1}{\mu} \nabla \wedge \mathbf{B}_{I}$, thus considering the looping currents near the walls (figure 11) which have an opposing effect on flow retardation. When $H_{a}$ augments, the currents intensify and the recirculation effects are accentuated, consequently widening the gap between the two solutions. Of course, neglecting the looping currents, by no means imitates the case where currents exit the walls, it does not, therefore, compensate the disparity between the results of Gold and Kinouchi et al (figure 18). Note that even if $B_{I}$ is very small, there will always be non negligi- 
ble current loops, since they are related to the curl of $B_{I}$ by the factor $\frac{1}{\mu}$. Despite the fact that the approximate solution neglects the effect of these loops on the flow, it has to acknowledge the presence of a return path for the considered current. If the path did not exist, the charges would accumulate and the induced electric field would end up compensating the Lorentz force, and so the charge separation would stop, and the flow would return to normal. Figure 18 plots the same data as figure 5 in Gold's paper and figure 4 given in the paper of Keltner et al. The curves start at $\frac{\overline{\tilde{U}}}{G}=\frac{1}{8}$ for $H_{a}=0$, which matches exactly the Poiseuille flow value.

\subsection{Induced potential}

Supposing that the electromagnetic steady state is instantly reached (see section 5), the charge conservation equation, $\nabla \cdot \mathbf{j}+\frac{\partial \rho_{e}}{\partial t}=0$, transforms into $\nabla \cdot \mathbf{j}=0$. Thus the divergence of Ohm's equation (5) gives,

$$
\nabla \cdot \mathbf{E}+\nabla \cdot(\mathbf{u} \wedge \mathbf{B})=0
$$

Likewise, neglecting time dependence in equation (2) implies that $\nabla \wedge \mathbf{E}=0$; hence $\mathbf{E}$ derives from an electric potential $\mathbf{E}=-\operatorname{grad} \phi$, and therefore,

$$
\Delta \phi=\nabla \cdot(\mathbf{u} \wedge \mathbf{B})
$$

If we assume that $\phi(r, \theta)=f(r) \sin \theta$, as done by [22], then (27) yields,

$$
\begin{aligned}
\sin \theta \frac{\partial^{2} f}{\partial r^{2}}+\frac{1}{r} \frac{\partial f}{\partial r} \sin \theta-\frac{f}{r^{2}} \sin \theta= & \frac{1}{r} \frac{\partial}{\partial r}\left(r u B_{0} \sin \theta\right) \\
& +\frac{1}{r} \frac{\partial}{\partial \theta}\left(u B_{0} \cos \theta\right)
\end{aligned}
$$

which can also be written as,

$$
\frac{\partial}{\partial r}\left(r^{2} f^{\prime}-r f\right)=B_{0} r^{2} \frac{\partial u}{\partial r}
$$

This equation may be solved to obtain an expression for $f$, nonetheless in this study we are only interested in calculating a potential difference across the wall, and not a voltage distribution in the vessel.

Integrating (28) between 0 and $a$, using the boundary condition $u(a)=0$, gives,

$$
a^{2} f^{\prime}(a)-a f(a)=-B_{0} \bar{u} a^{2} .
$$

Since the walls are assumed to be insulating, the radial component of $\mathbf{j}$ is null for $r=a$, hence the projection on $\mathbf{e}_{\mathbf{r}}$ of Ohm's equation gives,

$$
\left(\nabla \phi \cdot \mathbf{e}_{r}\right)_{r=a}=0 \Rightarrow f^{\prime}(a)=0 .
$$

and thus we get $f(a)=B_{0} a \bar{u}$.

The maximum potential is attained for $\theta=\pi / 2$,

$$
\phi(a, \pi / 2)=B_{0} a \bar{u},
$$

that's what Kinouchi et al. call estimated potential from the electromotive force.

The voltage across the walls would then be given by,

$$
V=\phi(a, \pi / 2)-\phi(-a, \pi / 2)=2 B_{0} a \bar{u} .
$$

With $V_{0}=2 u_{0} B_{0} a$, we write (30) in a non-dimensional form using (26),

$$
\tilde{V}=\overline{\tilde{U}}=2 \frac{G}{H_{a}^{2}}\left(\frac{1}{2}-\frac{I_{1}\left(H_{a}\right)}{H_{a} I_{0}\left(H_{a}\right)}\right) .
$$


Figure 19 plots the induced voltages across the vessel walls computed using the exact and approximate solution. For small values of $H_{a}$, the curves coincide well. At $8 T$ for example $\left(H_{a}=0.9\right)$, the two calculations differ only by $9 \%$, and for $H_{a}=1.2\left(B_{0}=10.7 T\right)$, the error amounts to $12.5 \%$. On the other hand, for stronger static fields, the curves diverge rapidly because of the additionnal flow retardations introduced by Vardanyan's approximate solution. Beyond 15T, the difference starts exceeding $25 \%$.

\section{Discussion}

The electromagnetic state In the studied stationary flow cases, the electromagnetic process can also be presumed steady. In fact, the temporal variations of the electromagnetic quantities are defined by the charges dynamics, which are characterized by

$$
\begin{aligned}
\frac{\partial \rho_{e}}{\partial t} & =-\nabla \cdot \mathbf{j} & (\text { charge conservation) } \\
& =-\sigma \nabla \cdot \mathbf{E}-\sigma \nabla \cdot(\mathbf{u} \wedge \mathbf{B}) & \text { (by Ohm's law) } \\
& =-\frac{\sigma}{\epsilon} \rho_{e}-\sigma \nabla \cdot(\mathbf{u} \wedge \mathbf{B}) & \text { (by Maxwell's (4)) }
\end{aligned}
$$

giving,

$$
\frac{\partial \rho_{e}}{\partial t}+\frac{\sigma}{\epsilon} \rho_{e}=-\sigma \nabla \cdot(\mathbf{u} \wedge \mathbf{B})
$$

Since the time constant associated with this differential equation, i.e. $\tau=\frac{\epsilon}{\sigma} \approx 1.7 \cdot 10^{-11} s$, is very small, it would be legitimate to suppose that $\rho_{e}$ adapts instantly to the second member oscillations. Hence, we can assume that $\rho_{e}=-\epsilon \nabla \cdot(\mathbf{u} \wedge \mathbf{B}) \Leftrightarrow \frac{\partial \rho_{e}}{\partial t}=0$, which means that the charge density immediately attains steady state. Therefore, the electromagnetic fields, which are induced by the charge separation motion, also instantly stabilize and their time derivatives can be neglected.

Notes on the series convergence In order to correctly evaluate the velocity and inductions expressions containing series of decreasing terms, it was fundamental to determine a summation stopping point $\mathrm{N}$, beyond which the added terms would no longer significantly modify the sum result. For this purpose, we calculated normalized mean errors between the results obtained while summing $\sum_{0}^{N_{1}}$ and those obtained using the sum $\sum_{0}^{N_{2}}$, defined as,

$$
N M E\left(N_{1}, N_{2}\right)=\frac{1}{I} \sum_{i=1}^{I}\left|\frac{\left(q_{(N 1)}\right)_{i}-\left(q_{(N 2)}\right)_{i}}{\left(q_{(N 2)}\right)_{i}}\right|
$$

For the considered function $q$ (representing $\left.\tilde{U}, \tilde{B}_{I}, ..\right)$, obtained with either $N_{1}+1$ or $N_{2}+1$ terms, mean errors are computed for each space discretization point $i$, then averaged over all points $I$. A low $N M E\left(N_{1}, N_{2}\right)$ error, would imply that the terms between $N_{1}$ and $N_{2}$ can be safely disregarded, and if $N_{2}>>N_{1}$, then it would be reasonable to assume that the sum $\sum_{0}^{N_{1}}$ is a good estimate of $\sum_{0}^{\infty}$

Error calculations for the series defining the velocity (14), the induced magnetic field (15) (presenting the same kind of sum as $\tilde{U})$, the mean velocity $\overline{\tilde{U}}(16)$ and $\tilde{E}_{r}(\tilde{r}, \pi / 2)$ (22) have shown that the number of terms necessary for series convergence depends on the value of $H_{a}$ and increases with it. For $\tilde{U}(\tilde{r}, 0)$, for example, with $H_{a}=0.2$ the convergence is very fast and is achieved with only 5 terms. The improvement obtained by going from $N=5$ to 50 is very minor, we have $N M E(5,50)=7.3 \cdot 10^{-8}$. On the other hand, for $H_{a}=20$ we have to include 29 terms in the 
sum to attain that same precision order $(N M E(29,50)=$ $\left.3.2 \cdot 10^{-8}\right)$. It was also noted that in the plane $\theta=\pi / 2$ less terms are necessary than for $\theta=0$. For example, for $H_{a}=20$, with 29 terms in the plane $\pi / 2$ we have $N M E(29,50)=1.6 \cdot 10^{-11}$.

As for the series intervening in the expression of $\overline{\tilde{U}}$, it converges earlier than the other series. At $H_{a}=0.2$, just two terms suffice $\left(N M E(2,50)=3.6 \cdot 10^{-7}\right)$, and at $H_{a}=20$ we have $N M E(20,50)=2 \cdot 10^{-10}$. The same applies, for $\tilde{E}_{r}(\tilde{r}, \pi / 2)$ where we have $N M E(12,50)=4 \cdot 10^{-8}$ for $H_{a}=20$.

Thus, in order to compute the results presented previously we deemed adequate to set $N=30$, given that the computations don't go beyond $H_{a}=20$.

\section{Some inconsistencies with Gold's plots Some numerical} results computed with Matlab using Gold's solution have shown discordances with those presented in Gold's paper:

i- The plots in figure 4 are identical to those of figures 1 and 2 shown by Gold [17], with only a small disparity that can be perceived in the plot where $\theta=0$ and $H_{a}=1.2(\alpha=0.6$ according to Gold's notation $)$. Note that in Gold's paper this curve differs from those with higher $H_{a}$, and presents an abrupt slope change, the kind that would be obtained when computing the series with an insufficient number of terms.

ii- The plot $V / V_{0}$ in figure 16 represents the sensitivity as defined by Gold, it traces the same curve shape as in Gold's figure 4 plotted from an integral expression; nonetheless, the obtained values here are not ex- actly identical. Our integral calculations of $\tilde{E}_{r}(\tilde{r}, \pi / 2)$ have been verified using two distinct numerical methods (the trapeze method and Simpson's quadrature method), which are supposed to work properly given the regularity of the function at hand (figure 20). Moreover, the obtained $\tilde{E}_{r}$ values were double-checked, by computing them from (22), as well as using numerical differentiations of $\tilde{B}$, which values coincide well with Gold's.

These inconsistencies might be explained by the accuracy differences between the calculations obtained in the early sixties using limited computational techniques, and those given by current high precision modern tools.

Induced potential on the thorax As explained in the introduction, when the body is subjected to a static magnetic field, magnetohydrodynamic voltages get superimposed on the ECG signal, altering its waveform. In fact, the potentials generated across the vessel walls by the electrodynamic interactions of the static field with arterial blood flow propagate to the surface of the thorax and are detected by the ECG sensors. During MRI examinations, the largest potentials are induced in the aortic arch, since it is perpendicular to the magnetic field, and presents the highest flow rate (figure 21(a)). Actually, the major magnetohydrodynamics-caused change observed on the ECG is an increase of the T wave (ventricle repolarization wave) which coincides with the blood ejection phase in the aorta.

The hypothesis of insulating vessel walls here rules out 
the possibility of computing surface potential using conductions in the surrounding tissues as done by Kinouchi et al. [21]. Nonetheless, a rough estimation could be obtained using a simple electric dipole approximation. The charge separation might indeed be compared to an electric dipole, and the potential at a remote observation point $M$ can be estimated using $V(M)=\frac{\mathbf{p} \cdot \mathbf{O}^{\prime} \mathbf{M}}{4 \pi \epsilon\left|\mathbf{O}^{\prime} \mathbf{M}\right|^{3}}, O^{\prime}$ being the dipole center, and $\mathbf{p}$ the equivalent dipole moment of the charge distribution, $\mathbf{p}=\iiint \rho_{e} \cdot \mathbf{d} d \tau$, where $\mathbf{d}$ represents the distance of the elementary volume $d \tau$ to the origin reference point $\left(\equiv O^{\prime}\right)$. Focusing on the the aortic arch, and using the charge distribution density given in (19) we can compute the dipole moment as, $\mathbf{p}=\rho_{e_{0}} a^{3} L \pi \overline{\tilde{U}} \mathbf{e}_{y}$, oriented in the same direction as the charge separation.

If we assume that the distance from the arch center to the surface is much greater than the dipole dimensions, and that the surrounding tissues constitute a homogeneous space, then the potential at point $M$ given in figure 21(b) situated on the thorax at a distance $d$ from the arch center can be expressed as $V(M)=\frac{E_{0} a^{2} L \overline{\tilde{U}}}{4 d^{2}}$. For $B_{0}=1 T,[21]$ calculate an induced potential of $1.18 \mathrm{mV}$ in a point situated at $7 \mathrm{~cm}$ above the aorta, which is considered to be of infinite length, and estimate that for a $6 \mathrm{~cm}$ aorta this value would be reduced 2.5 times, which amounts to $0.47 \mathrm{mV}$. Even though the configuration here is not exactly identical to theirs, the mentioned point is equivalent to $M$ in figure $21(\mathrm{~b})$. Using a $6 \mathrm{~cm}$ aortic arch, the dipole approximation yields $V(M)=0.18 m V$. This 2.6 factor, would be essentially due to the wall conductivity, since Kinouchi et al. reported a 2.3 potential reduction factor when the conductivity is divided by 10 . Hence, by considering a finite length and using a low conductivity, the calculations of Kinouchi et al. would result in $0.2 \mathrm{mV}$ potential, compared to $0.18 m \mathrm{~V}$ given by the dipole approximation.

We could therefore conclude that despite the fact that the dipole method constitutes a very rough approximation, it might be useful to estimate a correct order of magnitude for the surface potential, using simple calculations.

For comparison with real experimental measurements, further considerations have to be made. Measured T wave elevations in a $1.5 T$ magnet have been noted to range from $0.2 m V$ to $0.7 m V$ [12]. Using a more realistic arch length of $4.5 \mathrm{~cm}$, the dipole approximation yields a potential $V(M)=0.2 m V$ (with the same order of magnitude as the $\mathrm{T}$ wave). However, these recorded values cannot be directly compared to the theoretical approximation since the measured potential is, in fact, a voltage difference between the electrodes sites of measurement. In order to compare the calculated values with the experimental $\mathrm{T}$ wave distortions, we should define a realistic geometry of the thorax, reproduce the positioning of the electrodes relatively to the aortic arch, and set a more accurate orientation of the latter. Furthermore, we should not neglect the fact that the measured values represent a maximum amplitude of a pulsed potential whereas calculation give a steady potential which would be comparable to the mean value of the pulsed potential. 
Periodic flows Unlike the steady flow case, an exact solution of the time dependent equations (12) is unattainable, since decoupling them is impossible. In fact, (12) written in matrix form is

$$
\begin{aligned}
\frac{\partial}{\partial \tau} \underbrace{\left[\begin{array}{cc}
R_{e} & 0 \\
0 & R_{m}
\end{array}\right]}_{M_{1}}\left[\begin{array}{c}
\tilde{U} \\
\tilde{B}
\end{array}\right] & =\left[\begin{array}{c}
G \\
0
\end{array}\right]+\Delta\left[\begin{array}{c}
\tilde{U} \\
\tilde{B}
\end{array}\right] \\
& +\left[\cos \theta \frac{\partial}{\partial \tilde{r}}-\frac{\sin \theta}{\tilde{r}} \frac{\partial}{\partial \theta}\right] \underbrace{\left[\begin{array}{cc}
0 & \frac{H_{a}^{2}}{R_{m}} \\
R_{m} & 0
\end{array}\right]}_{M_{2}}\left[\begin{array}{c}
\tilde{U} \\
\tilde{B}
\end{array}\right]
\end{aligned}
$$

We can clearly note that $M_{1}$ and $M_{2}$ do not commute, i.e. $M_{1} M_{2} \neq M_{2} M_{1}$. Consequently, their diagonalisation in the same basis is not possible, which means that no linear variable change using constant coefficients would allow decoupling.

Therefore, studying the non steady flow problem requires simplifications such as neglecting the induced fields. The problem would then be reduced to solving,

$$
\rho \frac{\partial u}{\partial t}=-\frac{\partial p}{\partial z}+\eta\left(\frac{1}{r} \frac{\partial u}{\partial r}+\frac{\partial^{2} u}{\partial r^{2}}\right)-\sigma u B_{0}^{2},
$$

in order to derive the velocity expression when a time dependent pressure gradient is applied.

Sud et al. [18] proposed a sinusoidal solution using $-\frac{\partial p}{\partial z}=$ $A e^{i \omega t}$. If we set a more general sinusoidal gradient, such as $-\frac{\partial p}{\partial z}=A_{0}+A_{1} \cos \omega t$, we could compute a velocity profile, by applying successive Laplace and Hankel transforms to equation (34). We get the velocity expression,

$$
\begin{aligned}
u_{\sin }(\tilde{r}, t) & =2 \frac{a^{2}}{\eta} \sum_{n=1}^{\infty} \frac{J_{0}\left(\lambda_{n} \tilde{r}\right)}{\lambda_{n} J_{1}\left(\lambda_{n}\right)}\left(\frac{A_{0}}{\lambda_{n}^{2}+H_{a}^{2}}\right. \\
& +\frac{A_{1}\left[\left(\lambda_{n}^{2}+H_{a}^{2}\right) \cos \omega t+\alpha^{2} \sin \omega t\right]}{\left(\lambda_{n}^{2}+H_{a}^{2}\right)^{2}+\alpha^{4}} \\
& \left.+e^{-h \omega t}\left\{\frac{A_{0}}{\lambda_{n}^{2}+H_{a}^{2}}-\frac{A_{1}\left(\lambda_{n}^{2}+H_{a}^{2}\right)}{\left(\lambda_{n}^{2}+H_{a}^{2}\right)^{2}+\alpha^{4}}-\frac{A_{0}+A_{1}}{\left(\lambda_{n}^{2}+H_{a}^{2}\right)}\right\}\right)
\end{aligned}
$$

with $h=\frac{\lambda_{n}^{2}+H_{a}^{2}}{\alpha^{2}}$, where the $\lambda_{n}$ are the zeros of $J_{0}(x), J_{0}$ and $J_{1}$ are the Bessel function of the first kind with order 0 and 1 , and $\alpha=a \sqrt{\frac{\omega \rho}{\eta}}$.

This solution remains however specific to sinusoidal flows. A generic expression of the velocity profile can be formulated as a sum over the Fourier coefficients of the pressure gradient $g_{k}$, as done by Abi Abdallah et al. [19]:

$$
u(\tilde{r}, t)=2 \sum_{k=-\infty}^{\infty} \sum_{n=1}^{\infty} \frac{J_{0}\left(\lambda_{n} \tilde{r}\right)}{\lambda_{n} J_{1}\left(\lambda_{n}\right)} \quad \frac{g_{k}}{i \omega_{k} \frac{a^{2}}{\nu}+\lambda_{n}^{2}+H_{a}^{2}} e^{i \omega_{k} t}
$$

with

$$
g_{k}=\frac{1}{T} \int_{0}^{T}-\frac{a^{2}}{\eta} \frac{\partial p(t, z)}{\partial z} e^{-i \omega_{k} t} d t
$$

and $\omega_{k}=k \frac{2 \pi}{T}$, where $T$ is the heart cycle period. This formula can be used for any periodic time-dependent pressure gradient, like for example a realistic arterial gradient derived from a windkessel lumped model as in [19].

All the above mentioned periodic flow results present the same limitations as Vardanyan's approximate solution, given that they make the same induction assumptions. In fact, solutions (35) and (36) yield velocities that oscillate around a mean value which is equivalent to Vardanyan's steady case velocity.

Note that a pulsed wall potential could be computed here by proceeding as in section 4.2 .

\section{Conclusion}

In this paper, we studied the steady magnetohydrodynamic flow of blood, in a rigid circular vessel with nonconducting walls. We reviewed the exact solution given 
by Gold [17], we plotted and interpreted velocity profiles, induced fields, currents and potentials. Moreover we showed the separation of charges in the vessel caused by the Lorentz force. Then, in order to examine the influence of inductions and the importance of wall conductivity, the results were compared with those obtained with other flow hypothesis. For this purpose, we presented an approximate solution that neglects induced fields, for which we calculated an induced voltage across the vessel wall, and we used the data of Kinouchi et al. [21] computed in a conducting vessel.

The application of an external magnetic field $B_{0}$ to a blood flow generates electromagnetic inductions in the fluid that reduce the flow rate and flatten the velocity profile while stretching it more prominently in the same direction as the applied field. These effects heighten when $B_{0}$ increases; the induced magnetic fields remain however very weak. Approximating the results by neglecting these induced fields, while assuming velocity profile symmetry, overestimates flow reductions. Even though the induced magnetic fields are insignificant, solving the problem while neglecting them is inexact. In fact, since $B_{I}$ intervenes in the equations multiplied by $1 / \mu$ which is also very small, its effect is not really negligible. Despite the inaccuracy of Vardanyan's approach, for low magnetic fields such as those used for MRI, it provides a good approximation of the results using relatively simple calculations. Moreover, in that case, wall potentials comparable to those of the exact solution can even be estimated.

On the other hand, making non-conducting walls assump- tion, produces a current recirculation inside the vessel, which presents a counter-effect on flow reduction. Flow rates computed using Gold's solution are therefore greater than those obtained by Kinouchi et al. using conducting walls. This, however, is not the case for the approximate solution that disregards the current loops and estimates lower flow rates. Likewise, induced potentials on Gold's insulating walls are overestimated in relation to the case where the currents can exit toward the surrounding tissues. Nevertheless, the results improvement provided by the conducting walls hypothesis are very minor when $H_{a}$ is small.

In summary, this study has shown that, for small Hartmann numbers, the effect of the vessel's conductivity is not significant, and that neglecting induced fields produces a very close approximation of Gold's exact solution. Hence, we conclude that in situations like MRI exposure the above mentioned simplifying assumptions remain quite reasonable.

\section{References}

1. C. Taylor, M. Draney, Annual Review of fluid Mechanics 36, 197 (2004)

2. O. Valiron, L. Peris, G. Rikken, A. Schweitzer, Y. Saoudi, C. Remy, D. Job, Journal of Magnetic Resonance Imaging $\mathbf{2 2 ( 3 ) , ~} 334(2005)$

3. T. Tenforde, Progress in Biophysics and Molecular Biology 87, $279(2005)$

4. J. Felblinger, J. Slotboom, R. Kreis, B. Jung, C. Boesch, Magnetic Resonance in Medicine 41, 715 (1999) 
5. S. Fischer, S. Wickline, C. Lorenz, Magnetic Resonance in Medicine 42, 361 (1999)

6. V. Vardanyan, Biofizika 18(3), 491 (1973)

7. J. Keltner, M. Roos, P. Brakeman, T. Budinger, Magnetic Resonance in Medicine 16, 139 (1990)

8. J. Tasu, E. Mousseaux, A. Delouche, C. Oddou, O. Jolivet, J. Bittoun, Magnetic Resonance in Medicine 44, 66 (2000)

9. C. Gaffey, T. Tenforde, Bioelectromagnetics 2, 357 (1981)

10. T. Tenforde, C. Gaffey, B. Moyer, T. Budinger, Bioelectromagnetics 4, 1 (1983)

11. A. Weikl, W. Moshage, D. Hentschel, R. Schittenhelm, K. Bachmann, Zeitschrift für Kardiologie 78(9), 578 (1989)

12. D. Abi Abdallah, V. Robin, A. Drochon, O. Fokapu, Alterations in human ECG due to the MagnetoHydroDynamic effect: A method for accurate $R$ peak detection in the presence of high MHD artifacts, in Proceedings of the 29th Annual International Conference of IEEE Engineering in Medicine and Biology Society, Lyon, France (2007), pp. $1842-1845$

13. P. Jehenson, D. Duboc, T. Lavergne, L. Guize, F. Gurin, M. Degeorges, A. Syrota, Radiology 166, 227 (1988)

14. D. Chakeres, K. Alayar, H. Bouboulas, D. Young, Journal of magnetic resonance imaging 18, 346 (2003)

15. A. Holden, Progress in Biophysics and Molecular Biology 87, $289320(2005)$

16. E. Korchevskii, L. Marochnik, Biofizika 10(2), 371 (1965)

17. R. Gold, Journal of Fluid Mechanics 13, 505 (1962)

18. V. Sud, P. Suri, R. Mishra, Studia biophysica 46(3), 163 (1974)

19. D. Abi Abdallah, V. Robin, O. Fokapu, A. Drochon, Computer Methods in Biomechanics and Biomedical Engineering 10(1), 159 (2007)
20. V. Sud, P. Suri, R. Mishra, Studia biophysica 69(3), 175 (1978)

21. Y. Kinouchi, H. Yamagushi, T. Tenforde, Bioelectromagnetics 17(1), 21 (1996)

22. R. Edgerton, Medical and Biological Engineering 6, 627 (1968) 
Table 1. Numerical values

\begin{tabular}{|c|r|r|}
\hline \multicolumn{3}{|c|}{ Blood parameters } \\
\hline$\eta$ & $4.10^{-3}$ & Pa.s \\
\hline$\rho$ & 1050 & $\mathrm{~kg} / \mathrm{m}^{3}$ \\
\hline$\sigma$ & 0.5 & $\mathrm{~S} / \mathrm{m}$ \\
\hline \multicolumn{3}{|c|}{ Vessel radius } \\
\hline$a$ & $10^{-2}$ & $\mathrm{~m}$ \\
\hline \multicolumn{3}{|c|}{ Flow characteristics } \\
\hline$u_{0}$ & 0.4 & $\mathrm{~Pa} / \mathrm{m}$ \\
\hline$\frac{\partial p}{\partial z}$ & -128 & $\mathrm{H} / \mathrm{m}$ \\
\hline & \multicolumn{3}{|c|}{ Medium characteristics } \\
\hline$\mu$ & $4 \pi 10^{-7}$ & $\mathrm{~F} / \mathrm{m}$ \\
\hline$\epsilon$ & $\frac{1}{(36 \pi)} 10^{-9}$ & \\
\hline
\end{tabular}

The typical characteristics of blood are used. The permeability and permittivity are assumed to be equal to those of free space.

The vessel radius is equivalent to that of aorta. The pressure gradient is one that would produce a Poiseuille flow with a mean velocity of $40 \mathrm{~cm} / \mathrm{s}$.

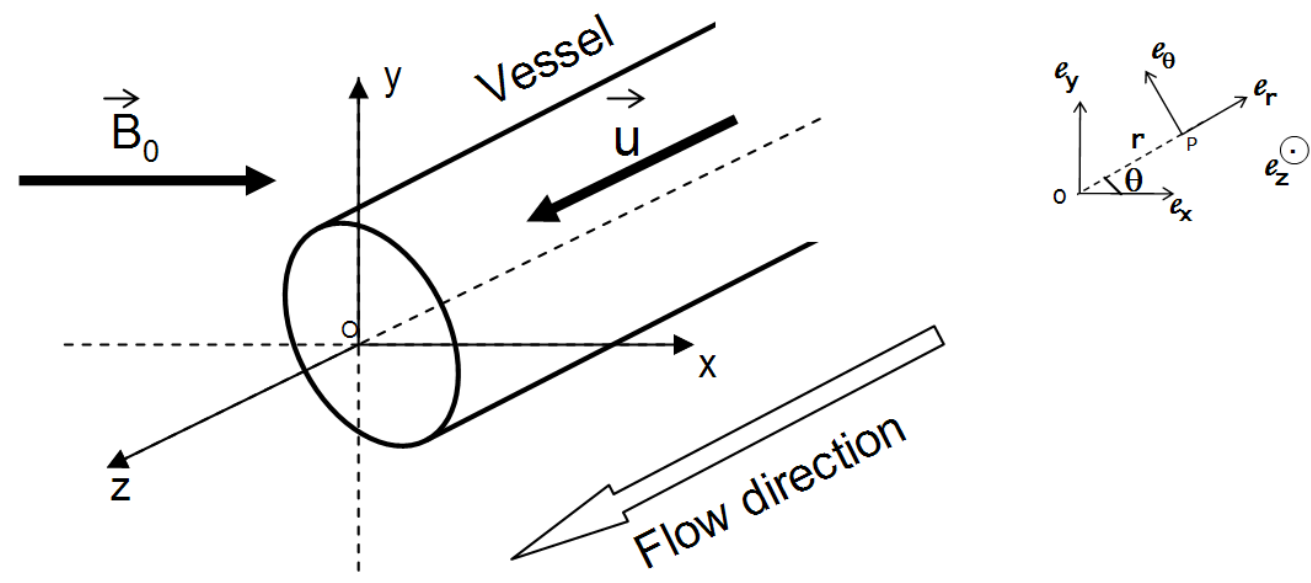

Fig. 1. The blood flows in the $\mathrm{Oz}$ direction, in the presence of a transverse static magnetic field oriented along the Ox axis. 


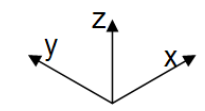

$\mathrm{U} / \mathrm{U} 0$ for $\mathrm{B} 0=0.0 \mathrm{~T}$

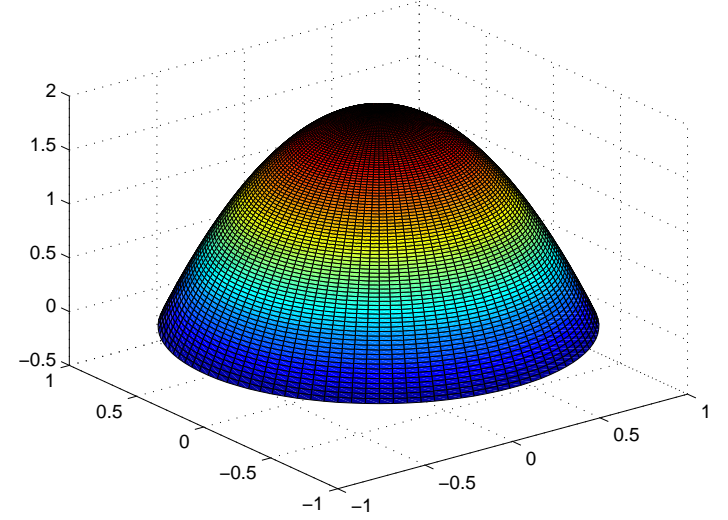

(a) $B_{0}=0 T\left(H_{a}=0\right)$

$\mathrm{U} / \mathrm{U} 0$ for $\mathrm{B} 0=8.0 \mathrm{~T}$

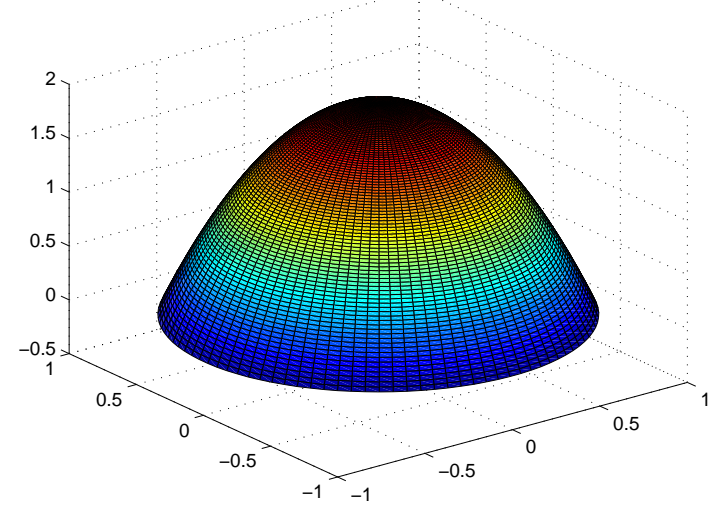

(c) $B_{0}=8 T\left(H_{a}=0.89\right)$

$\mathrm{U} / \mathrm{U} 0$ for $\mathrm{B} 0=30.0 \mathrm{~T}$

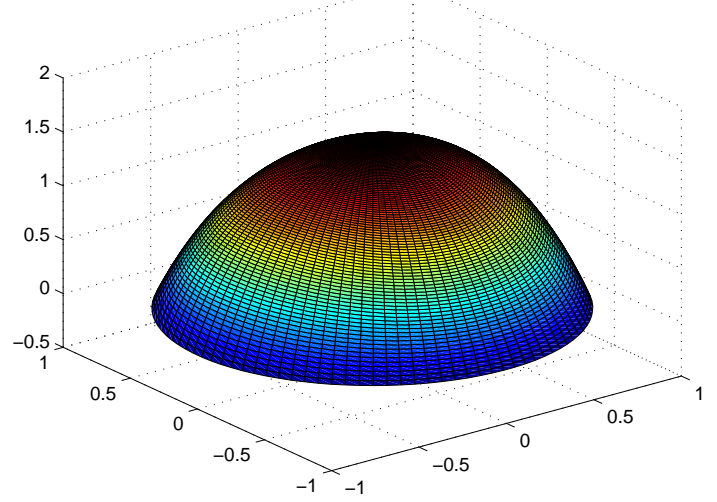

(e) $B_{0}=30 T\left(H_{a}=3.35\right)$
$\mathrm{U} / \mathrm{UO}$ for $\mathrm{B} 0=1.5 \mathrm{~T}$

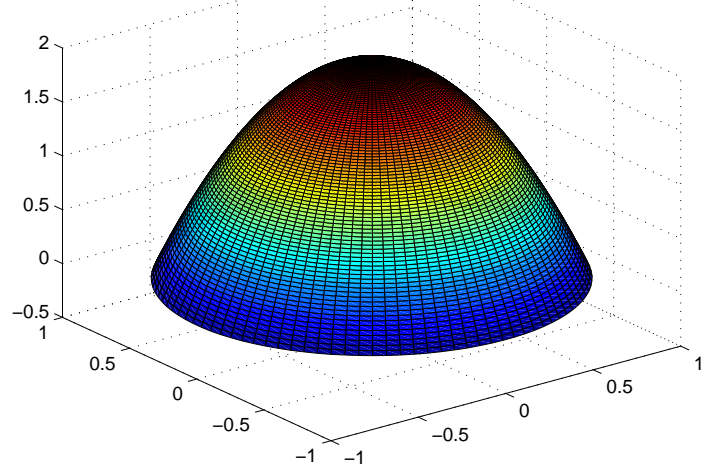

(b) $B_{0}=1.5 T\left(H_{a}=0.16\right)$

$\mathrm{U} / \mathrm{UO}$ for $\mathrm{B} 0=20.0 \mathrm{~T}$

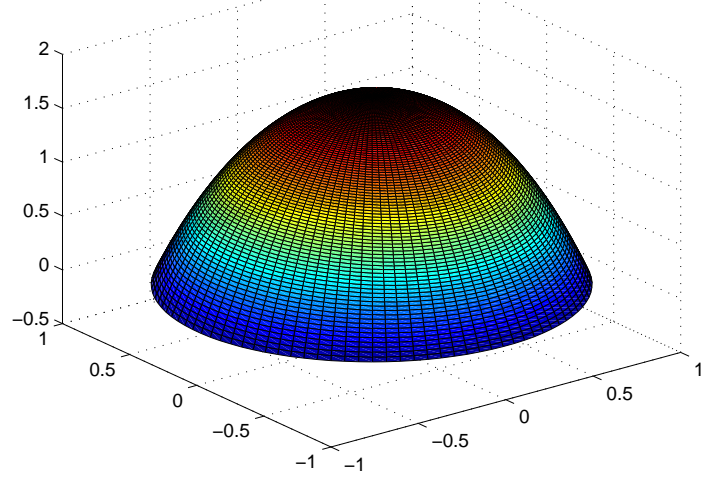

(d) $B_{0}=20 T\left(H_{a}=2.23\right)$

$\mathrm{U} / \mathrm{UO}$ for $\mathrm{B} 0=40.0 \mathrm{~T}$

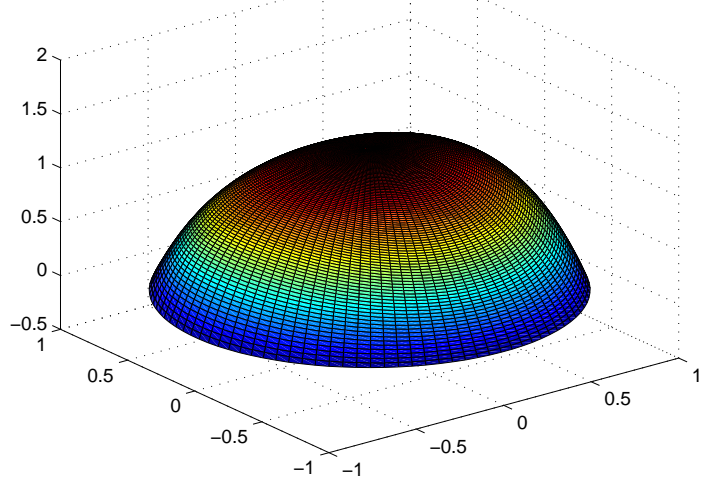

(f) $B_{0}=40 T\left(H_{a}=4.47\right)$

Fig. 2. Non-dimensional velocity profiles $u_{z} / u_{0}$ computed from (14) for various intensities of $B_{0}$. 

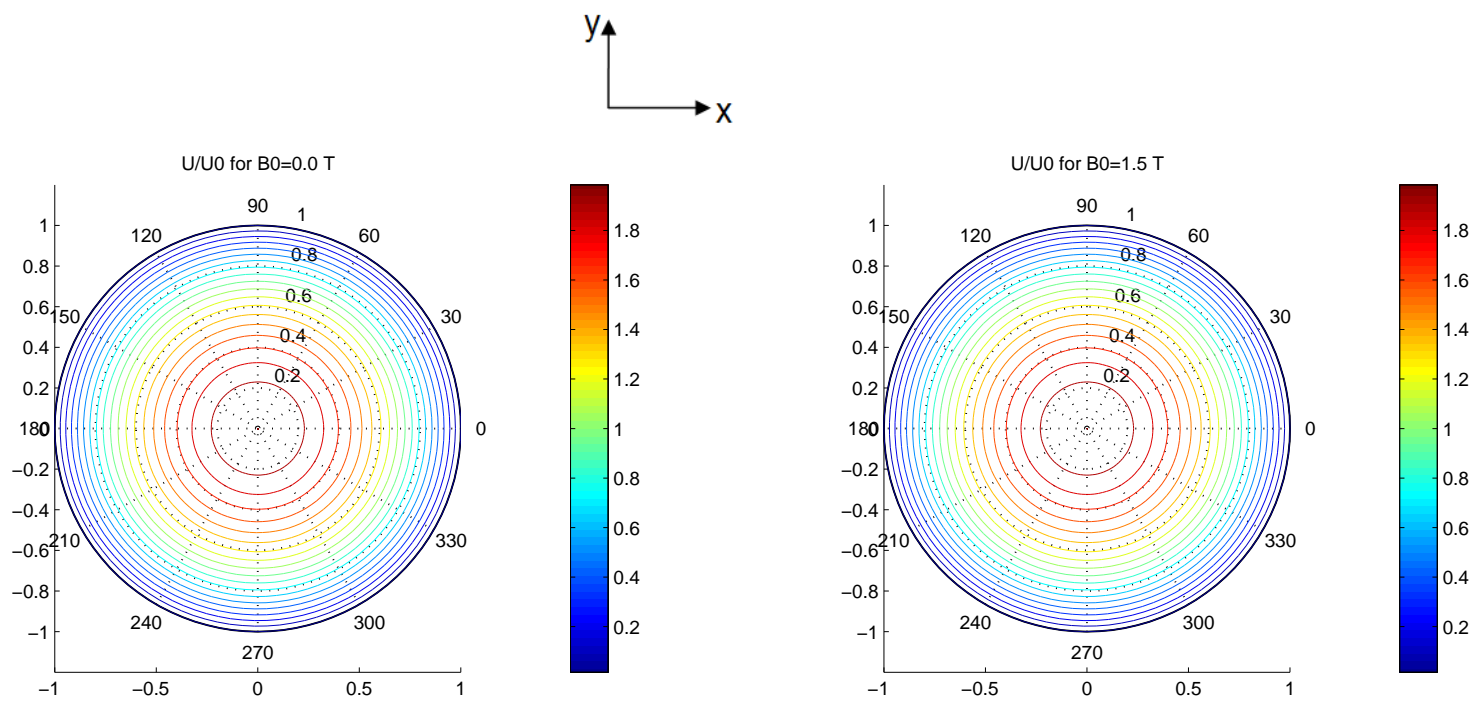

(a) $B_{0}=0 T\left(H_{a}=0\right)$

(b) $B_{0}=1.5 T\left(H_{a}=0.16\right)$
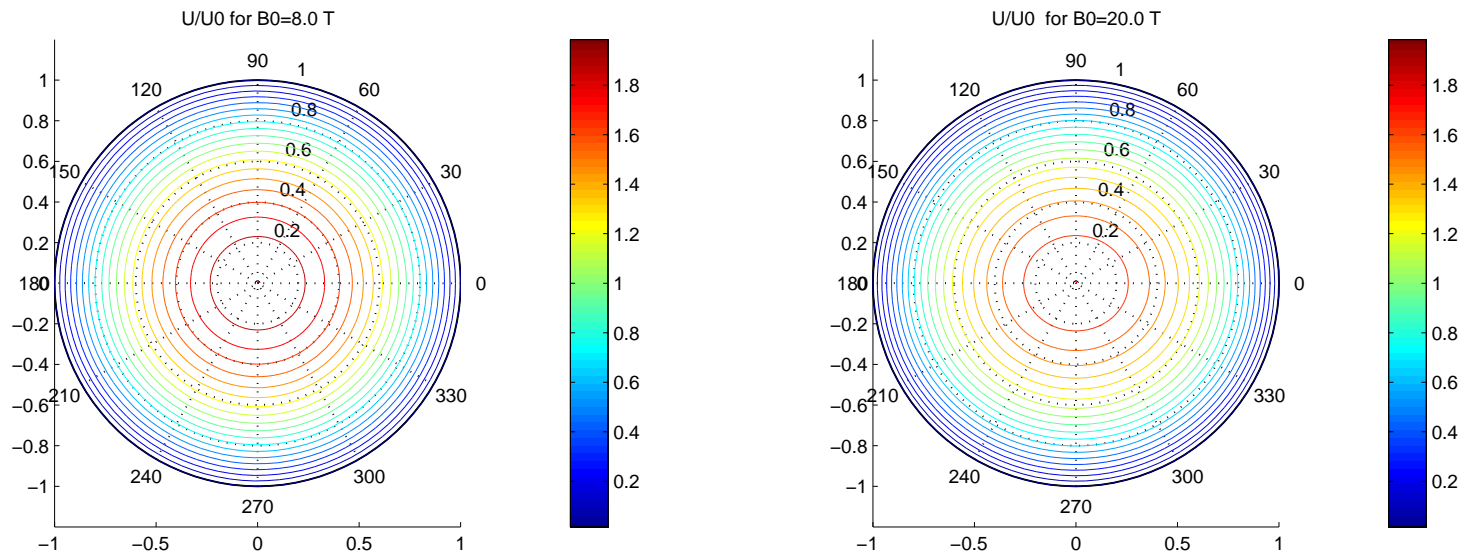

(c) $B_{0}=8 T\left(H_{a}=0.89\right)$

(d) $B_{0}=20 T\left(H_{a}=2.23\right)$
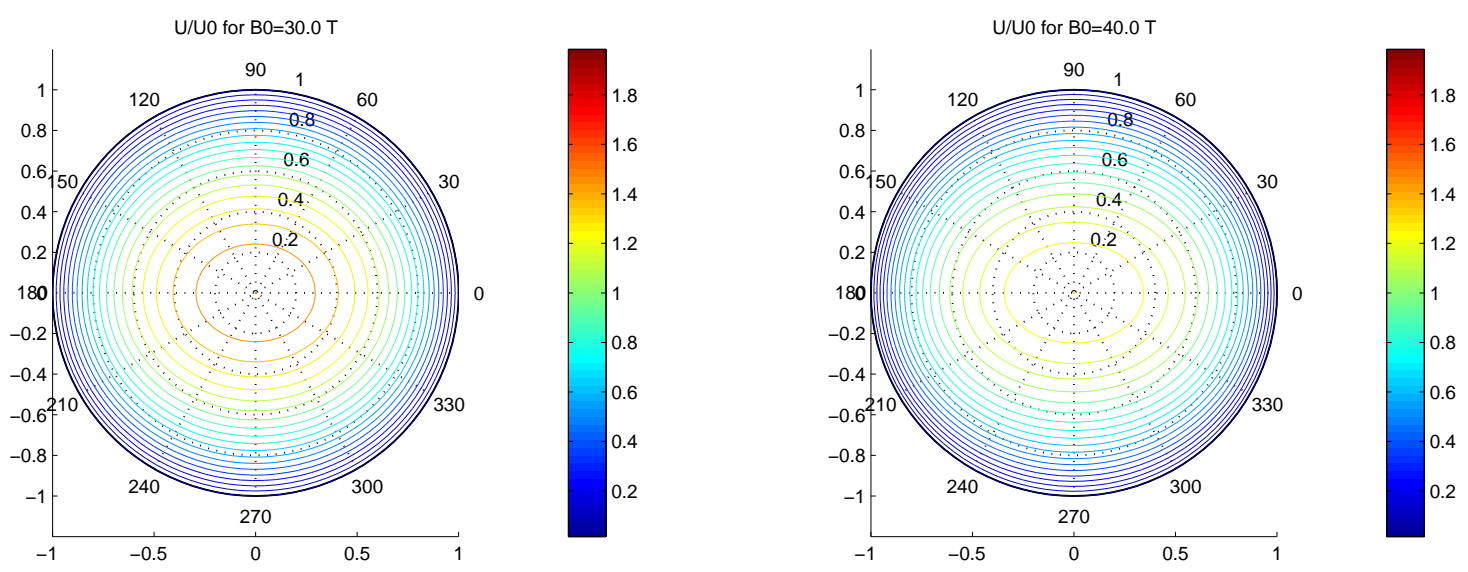

(e) $B_{0}=30 T\left(H_{a}=3.35\right)$

(f) $B_{0}=40 T\left(H_{a}=4.47\right)$

Fig. 3. Iso-velocity lines in the transverse plane, representing the amplitudes of the profiles given in figure 2 . 


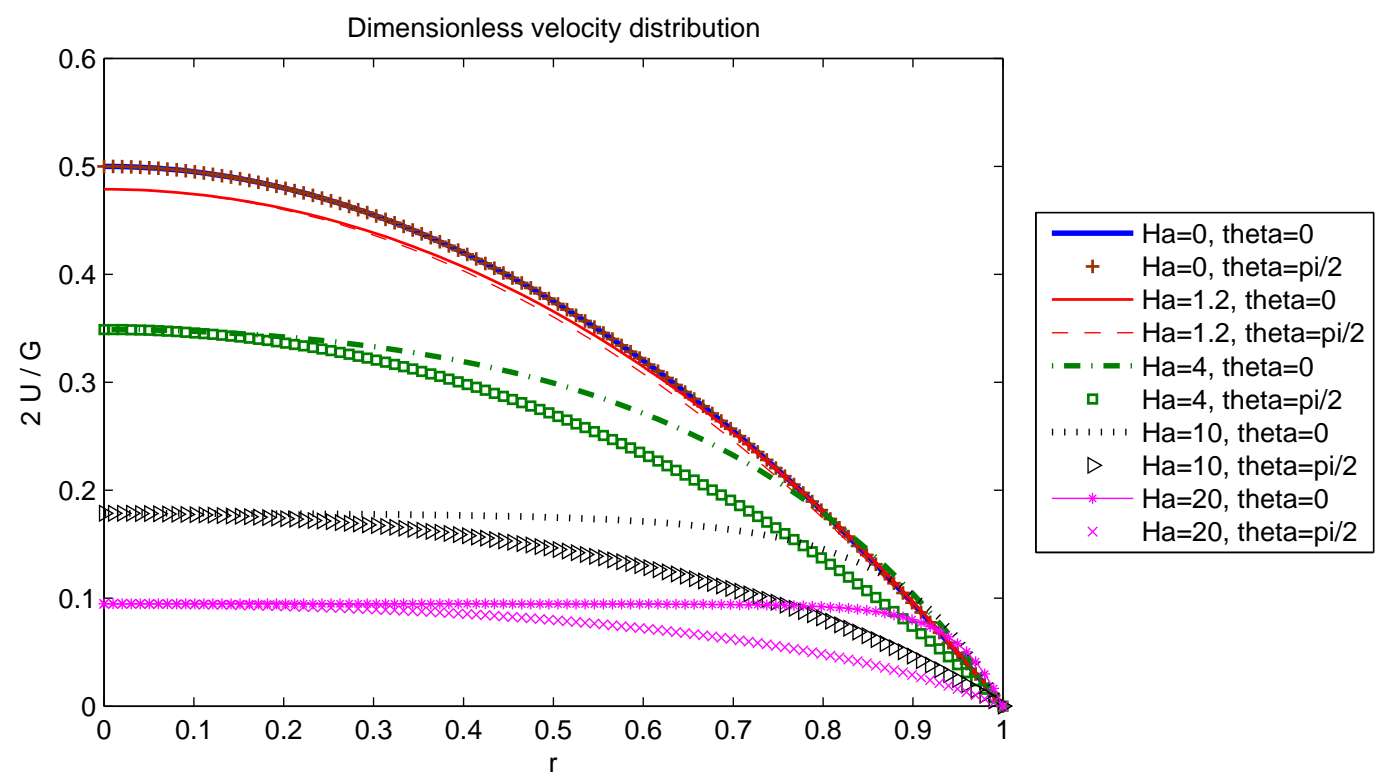

Fig. 4. Comparison of the non-dimensional velocity distribution $\frac{2 \tilde{U}}{G}$ computed from (14) and plotted in the planes $\theta=0$ and $\theta=\pi / 2$ for several values of $H_{a}$.

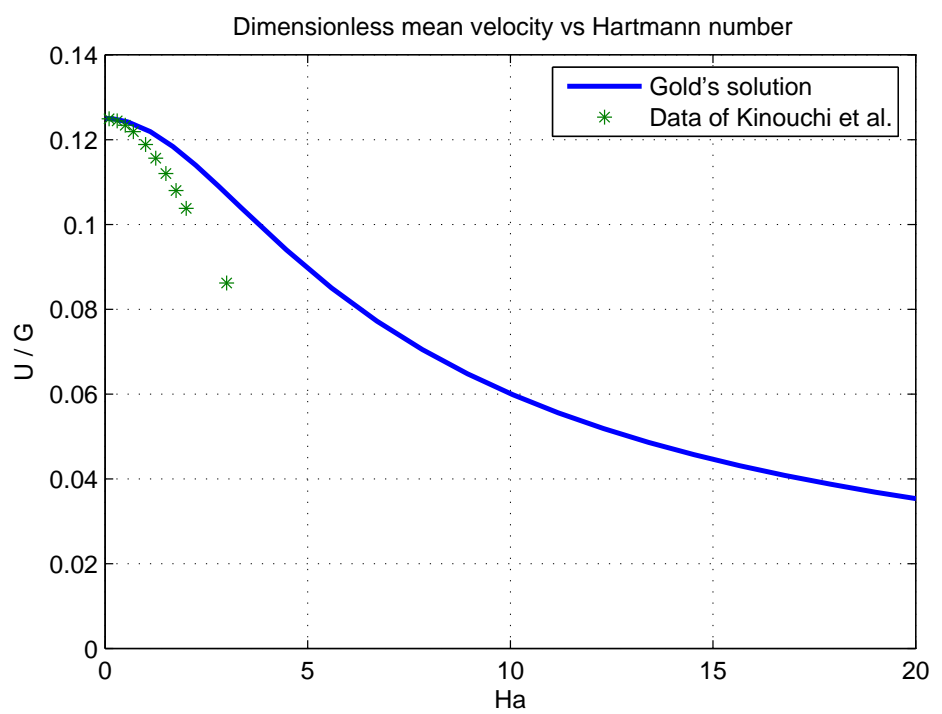

Fig. 5. Non-dimensional mean velocity $\frac{\bar{U}}{G}$ computed with (16) and compared to the values given by [21]. 


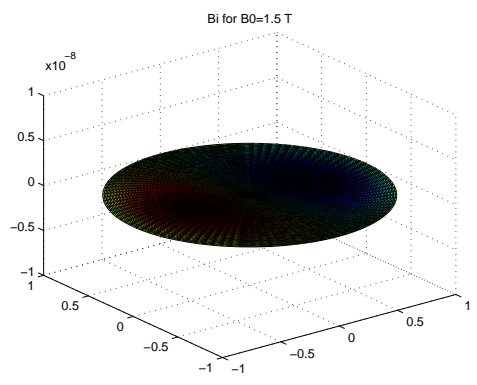

(a) $B_{0}=1.5 T\left(H_{a}=0.16\right)$

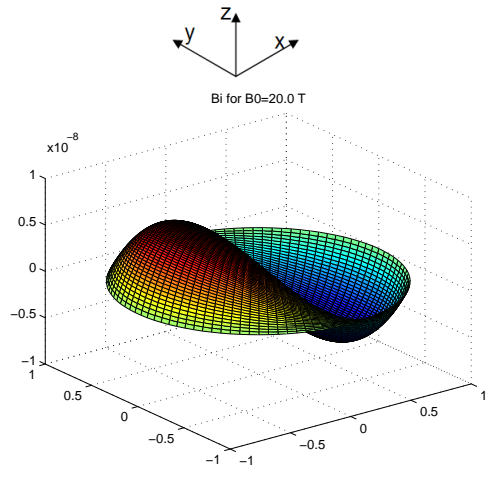

(b) $B_{0}=20 T\left(H_{a}=2.23\right)$

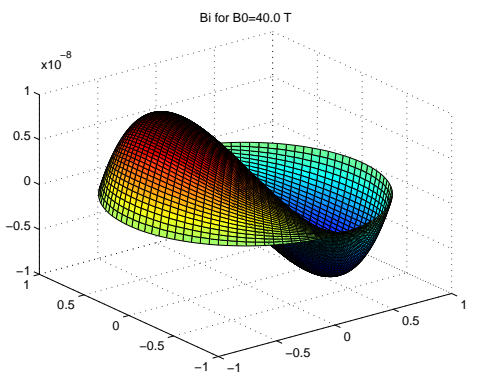

(c) $B_{0}=40 T\left(H_{a}=4.47\right)$

Fig. 6. Induced magnetic fields $B_{I} / B_{0}$ computed by (15) for various field intensities $B_{0}$ (graduation scale is $10^{-8}$ ).

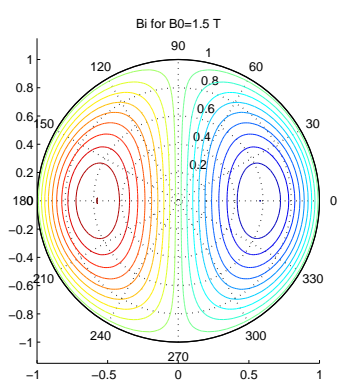

(a) $B_{0}=1.5 T\left(H_{a}=0.16\right)$

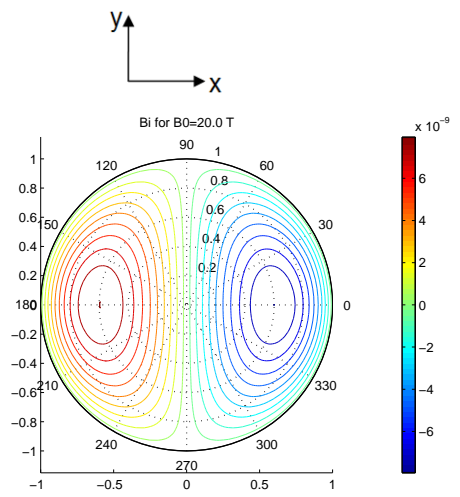

(b) $B_{0}=20 T\left(H_{a}=2.23\right)$

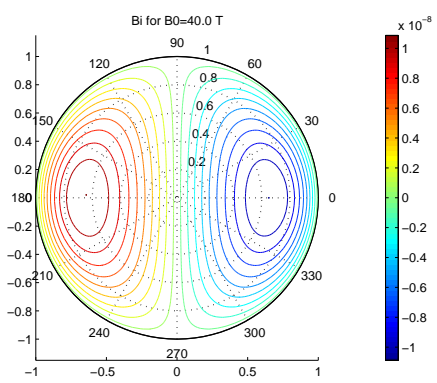

(c) $B_{0}=40 T\left(H_{a}=4.47\right)$

Fig. 7. Induced magnetic fields contours representing, in the transverse plane, the amplitudes of the field profiles shown in figure 6 (respective graduation scales are $10^{-10}, 10^{-9}$ and $10^{-8}$ )

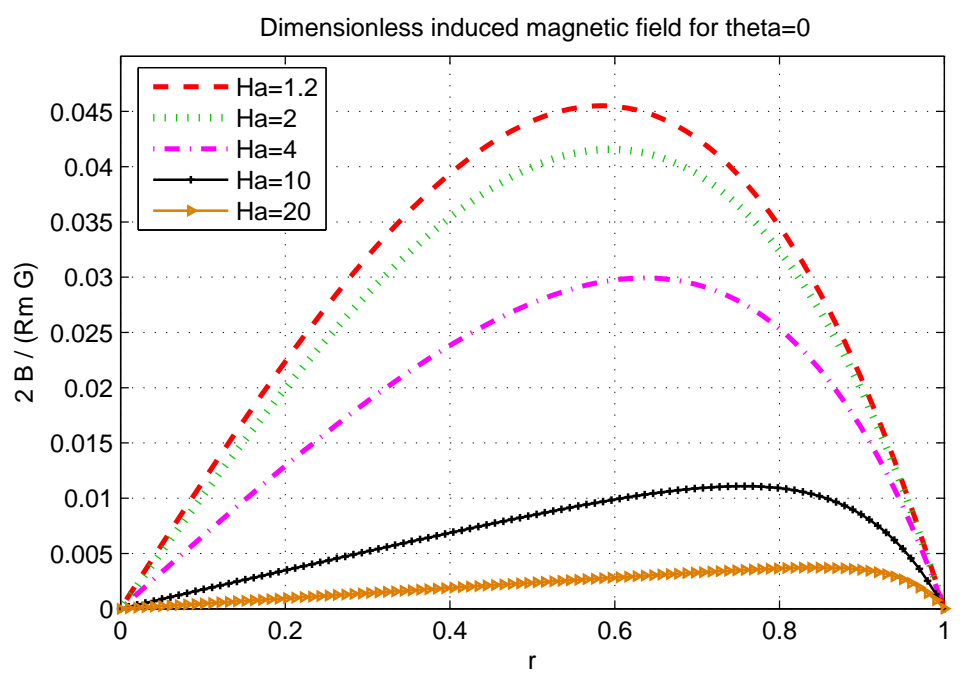

Fig. 8. Plots of Gold's number $\frac{-2 \tilde{B}}{R_{m} G}$ for $\theta=0$ 


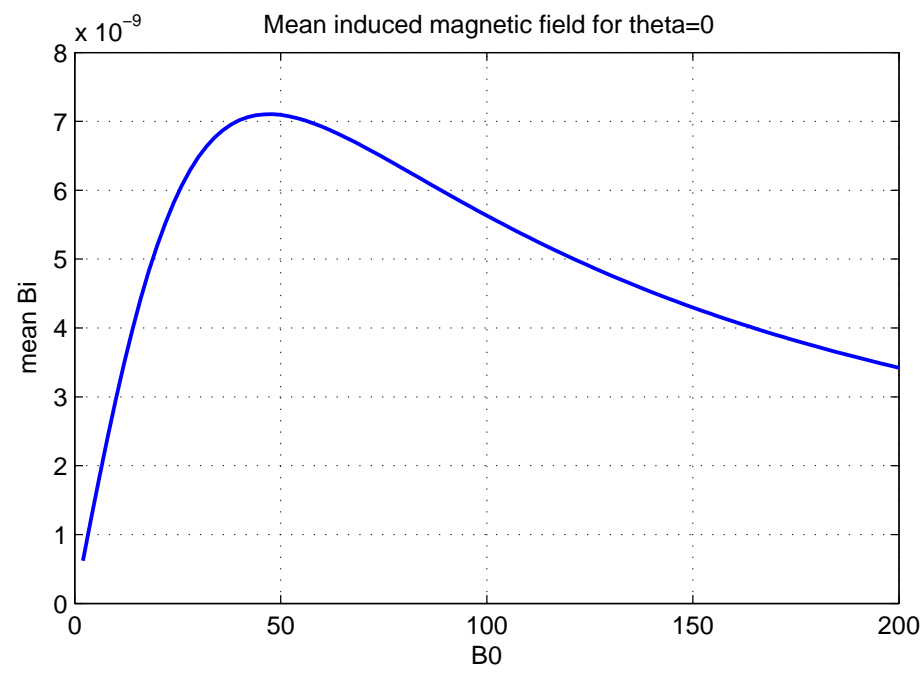

Fig. 9. Mean induced magnetic field intensity averaged over $r$, versus $B_{0}$ for $\theta=0$ (graduation scale is $10^{-9}$ Tesla).

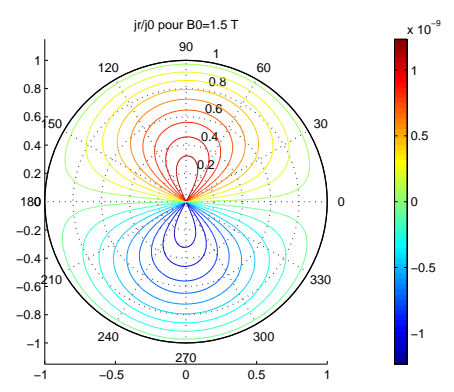

(a) $j_{r} / j_{0}$ for $B_{0}=1.5 T$

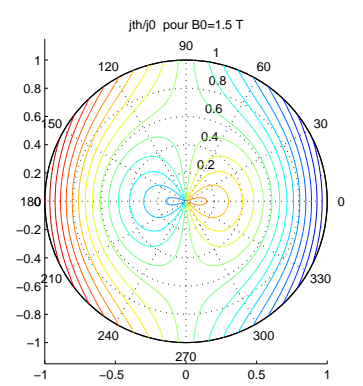

(d) $j_{\theta} / j_{0}$ for $B_{0}=1.5 T$
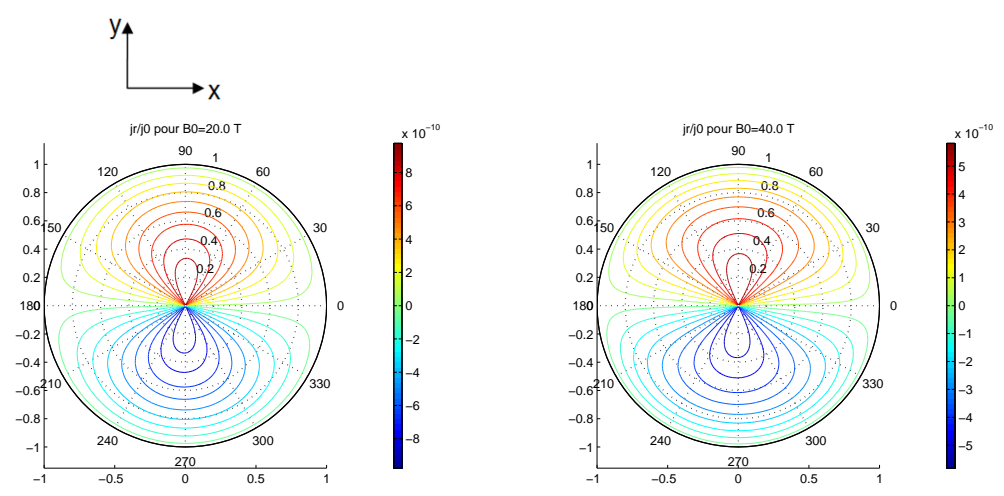

(b) $j_{r} / j_{0}$ for $B_{0}=20 T$

(c) $j_{r} / j_{0}$ for $B_{0}=40 T$

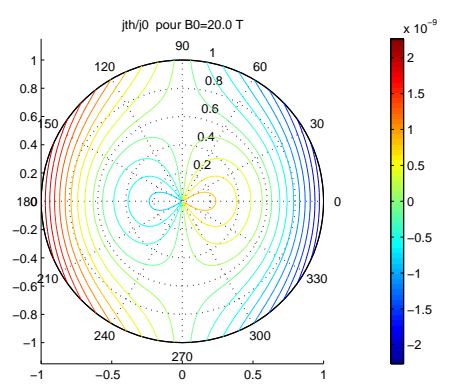

(e) $j_{\theta} / j_{0}$ for $B_{0}=20 T$

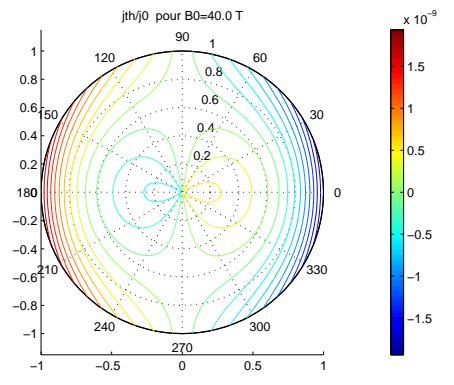

(f) $j_{\theta} / j_{0}$ for $B_{0}=40 T$

Fig. 10. Non-dimensional amplitudes of the transverse current density components, $j_{r} / j_{0}$ and $j_{\theta} / j_{0}$ computed from (17) and plotted for various intensities of $B_{0}$ (graduation scale is $10^{-9}$ for (a),(d),(e) and (f), and $10^{-10}$ for (b) and (c)). 


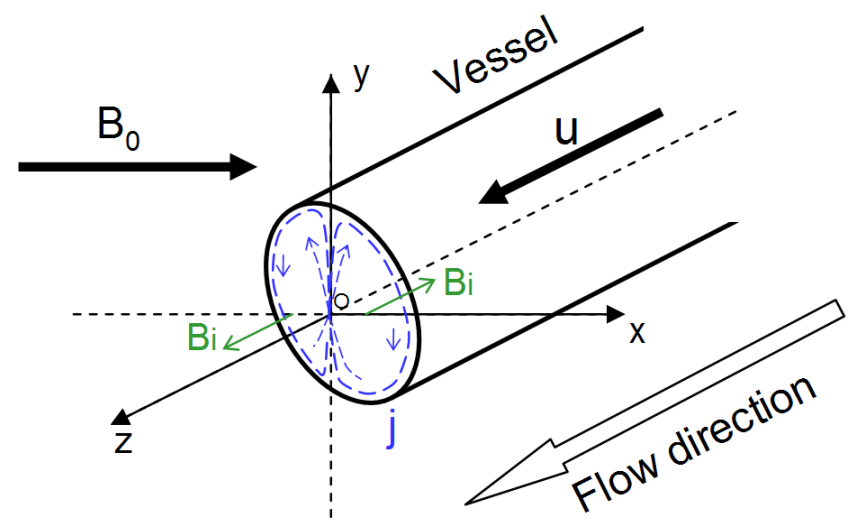

Fig. 11. The induced currents circulate in a closed loop in the transverse plane. The induced field is parallel to the $O z$ axis with opposite directions on each side of $O y$.

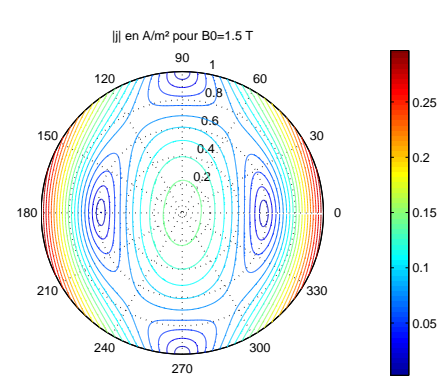

(a) $B_{0}=1.5 T\left(H_{a}=0.16\right)$

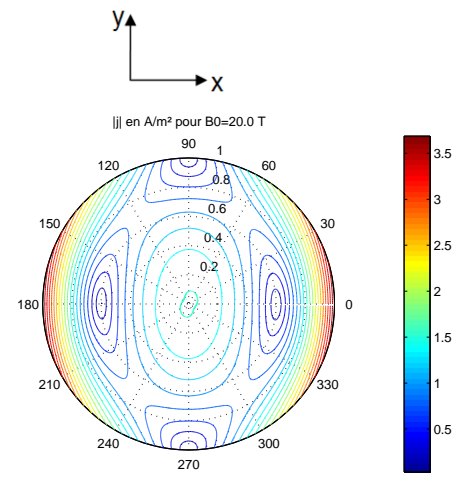

(b) $B_{0}=20 T\left(H_{a}=2.23\right)$

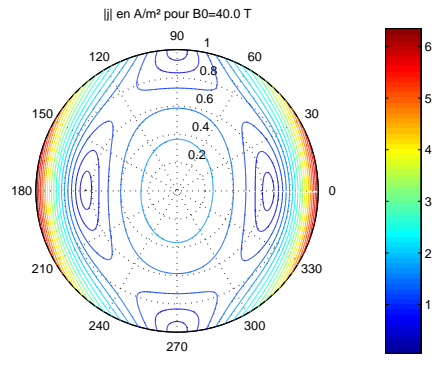

(c) $B_{0}=40 T\left(H_{a}=4.47\right)$

Fig. 12. Current density modulus $|j|=\sqrt{j_{r}^{2}+j_{\theta}^{2}}$ in $A / m^{2}$ for several values of $B_{0}$. 


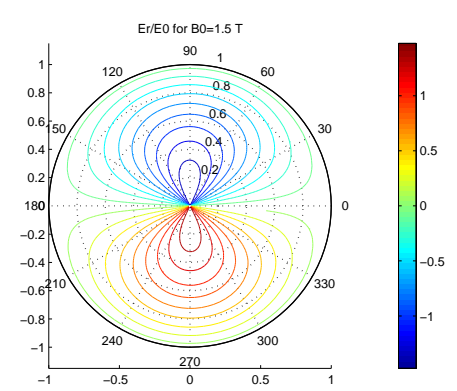

(a) $E_{r} / E_{0}$ for $B_{0}=1.5 T$

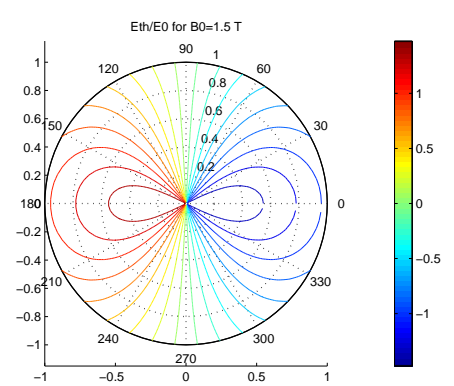

(d) $E_{\theta} / E_{0}$ for $B_{0}=1.5 T$

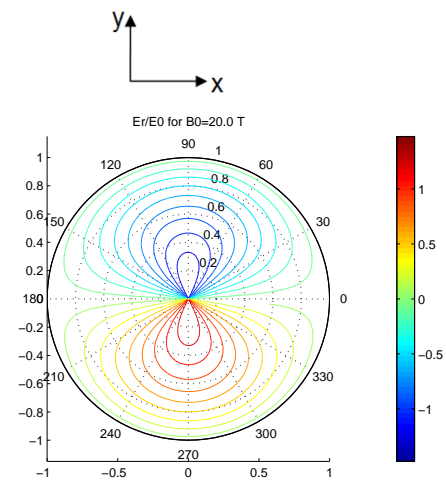

(b) $E_{r} / E_{0}$ for $B_{0}=20 T$

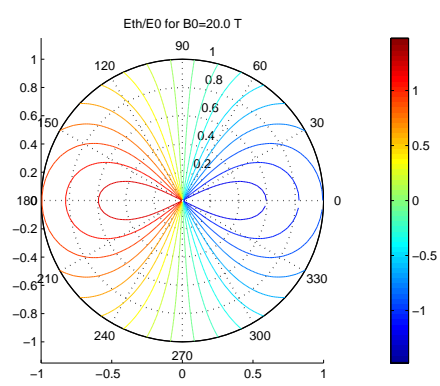

(e) $E_{\theta} / E_{0}$ for $B_{0}=20 T$

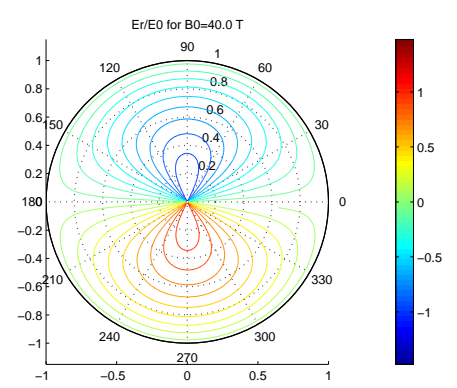

(c) $E_{r} / E_{0}$ for $B_{0}=40 T$

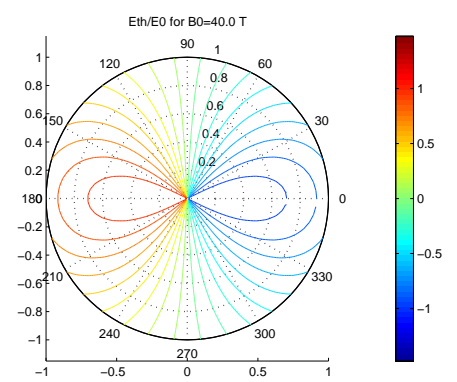

(f) $E_{\theta} / E_{0}$ for $B_{0}=40 T$

Fig. 13. Non-dimensional amplitudes of the induced electric field components, $E_{r} / E_{0}$ and $E_{\theta} / E_{0}$, computed from (18) and plotted for several values of $B_{0}$.

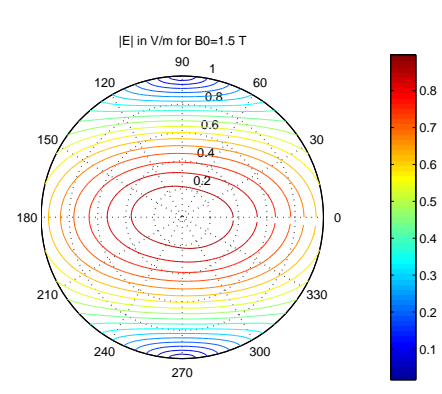

(a) $B_{0}=1.5 T\left(H_{a}=0.16\right)$

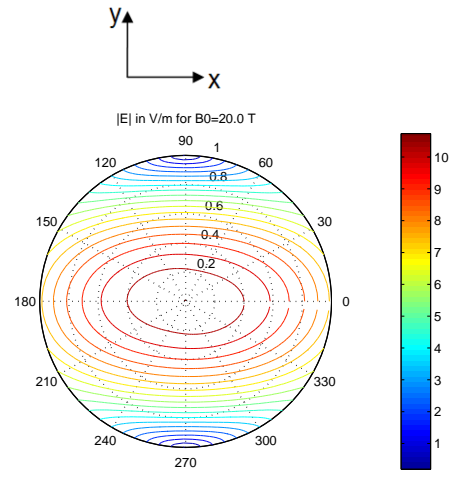

(b) $B_{0}=20 T\left(H_{a}=2.23\right)$

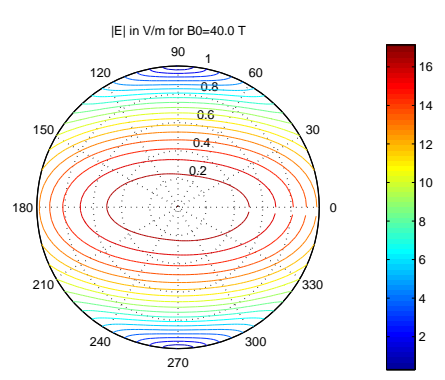

(c) $B_{0}=40 T\left(H_{a}=4.47\right)$

Fig. 14. Modulus of the induced electric field $|E|=\sqrt{E_{r}^{2}+E_{\theta}^{2}}$ in $V / m$ for several intensities of $B_{0}$. 


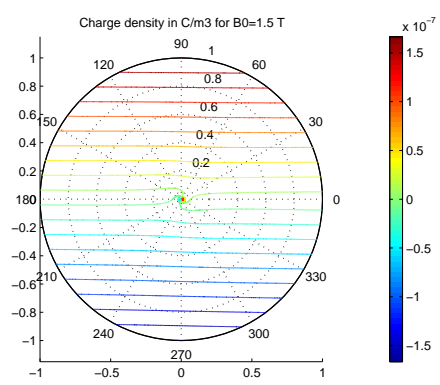

(a) $B_{0}=1.5 T\left(H_{a}=0.16\right)$
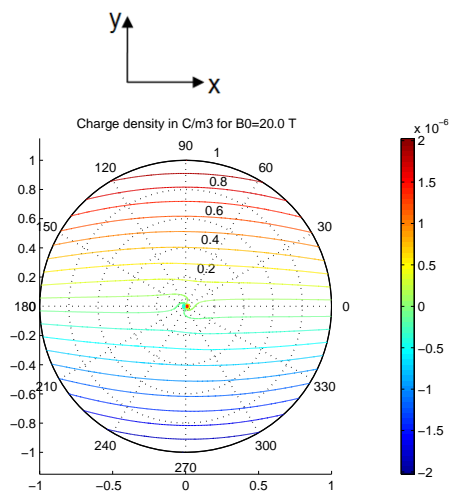

(b) $B_{0}=20 T\left(H_{a}=2.23\right)$

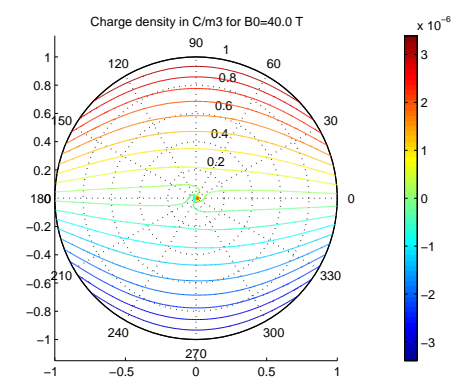

(c) $B_{0}=40 T\left(H_{a}=4.47\right)$

Fig. 15. Charge density in $C / m^{3}$ computed from (19) (graduation scales are $10^{-7}$ for (a) and $10^{-6}$ for (b) and (c))

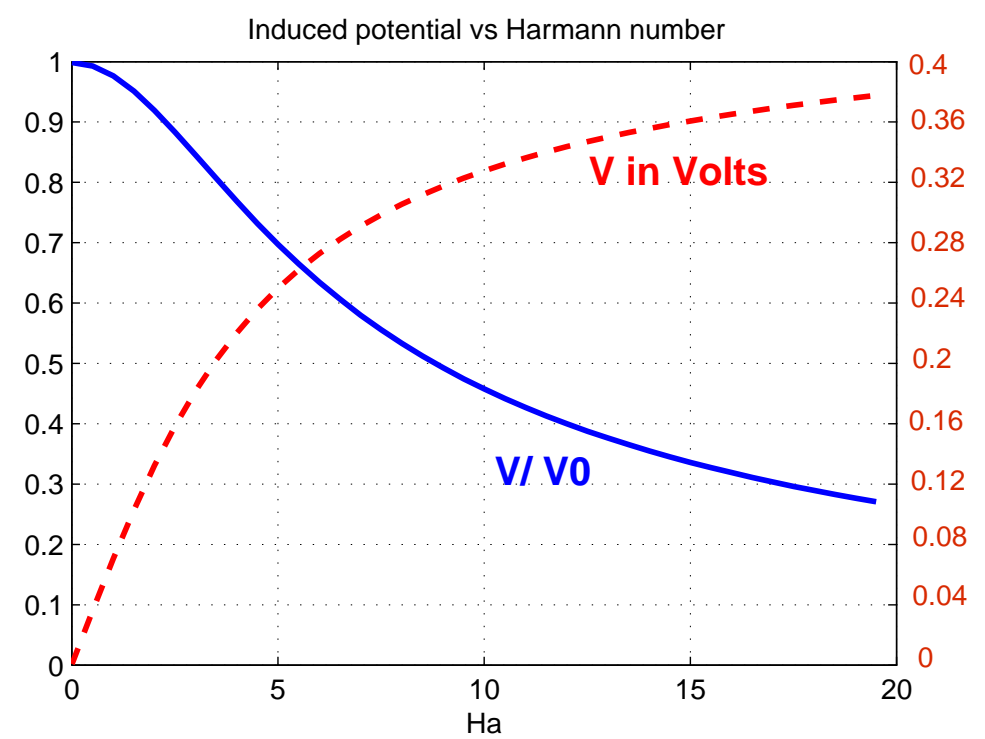

Fig. 16. Non-dimensional induced wall potential $\tilde{V}$ computed from (21) (left units) and $V=2 u_{0} B_{0} a \tilde{V}$ in $V$ olts (right units) 


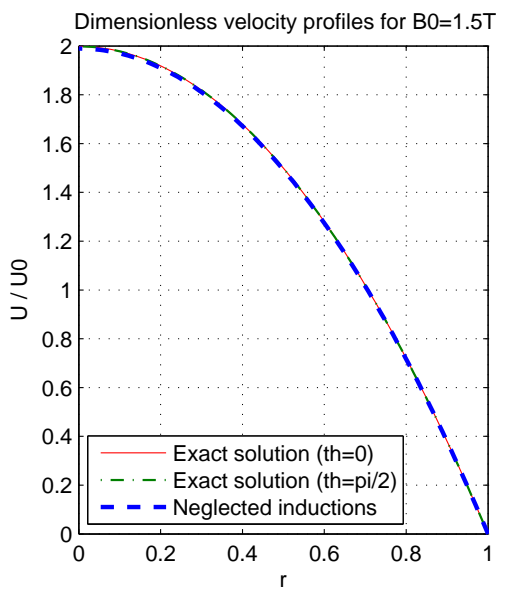

(a) $B_{0}=1.5 T$

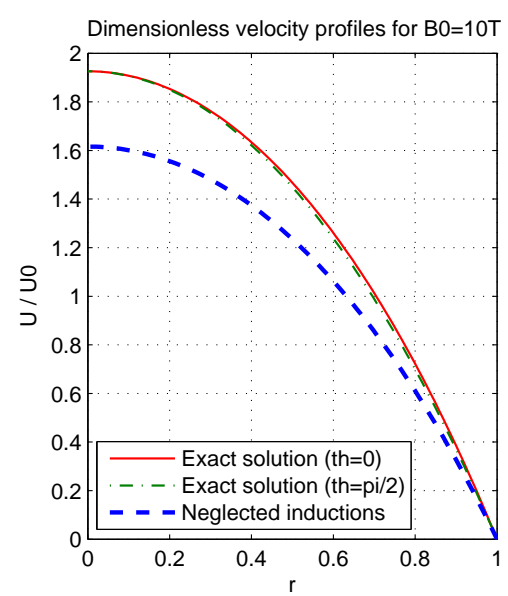

(b) $B_{0}=10 T$

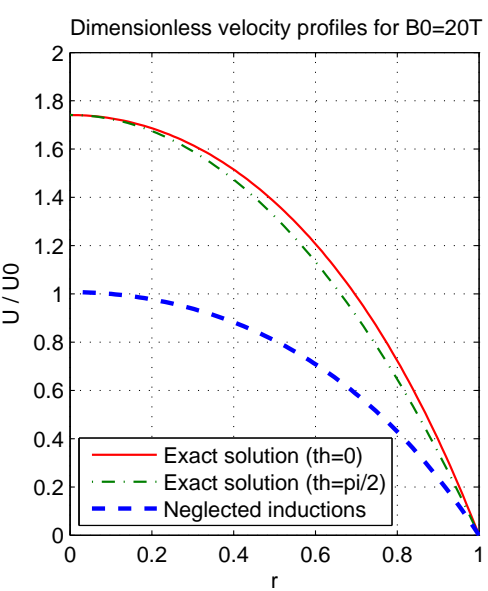

(c) $B_{0}=20 T$

Fig. 17. Comparison between Gold's exact solution velocity profile, computed from (14) for $\theta=0$ and $\theta=\pi / 2$, and Vardanyan's approximate solution (25) for $B_{0}=1.5 T, 10 T$, and $20 T$.

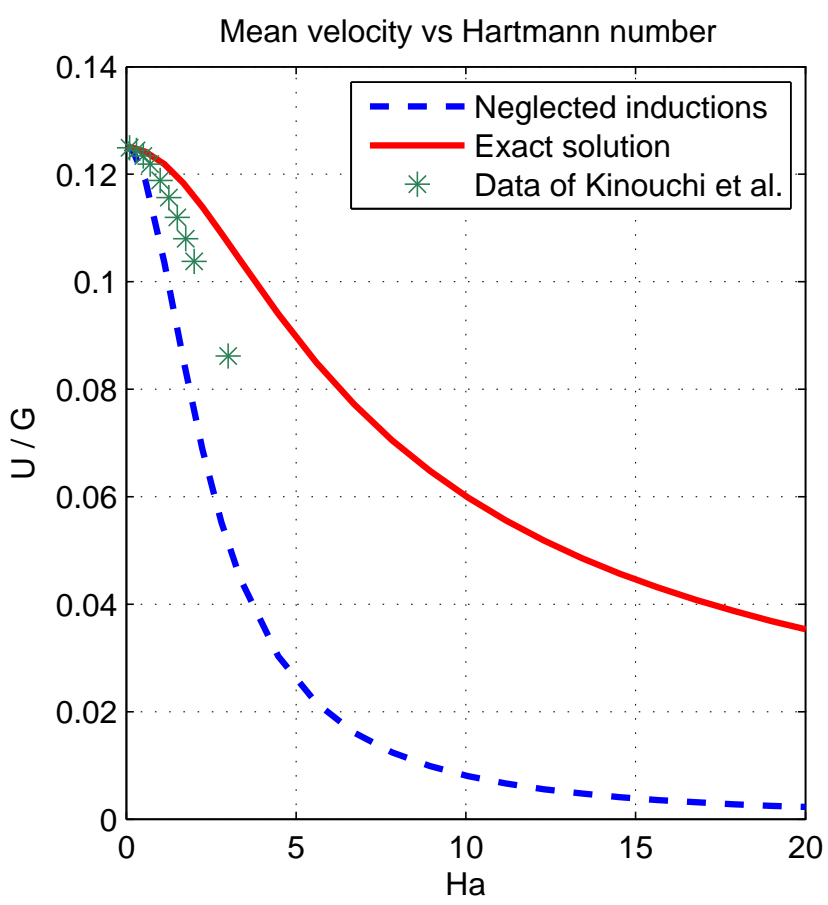

Fig. 18. Non-dimensional mean velocity $\frac{\bar{U}}{G}$. Comparison between Gold's exact solution mean velocity (16), the approximate solution mean velocity (26), and the data of Kinouchi et al. 


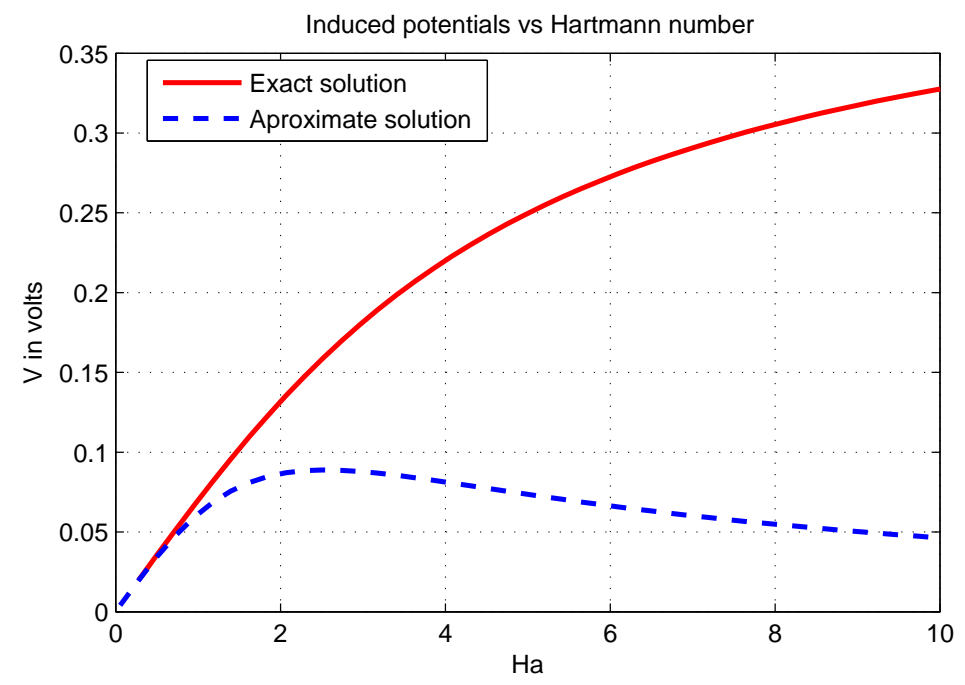

Fig. 19. Induced potentials across the insulating walls. Comparison between the induced voltages computed by the exact solution (21) and by the approximate solution (30).

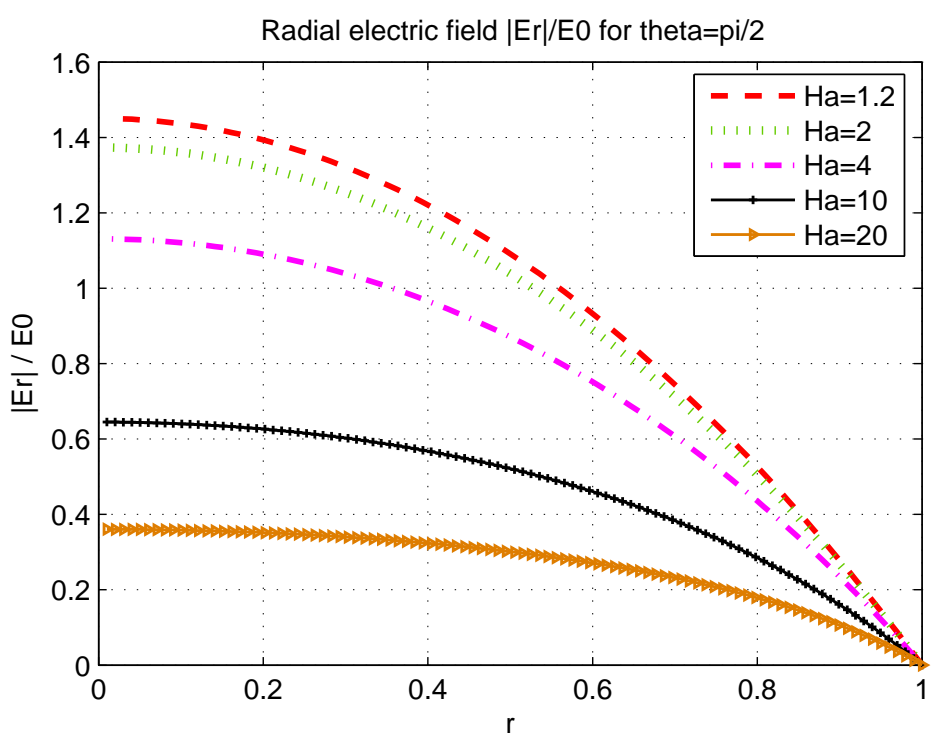

Fig. 20. Absolute value of the non-dimensional radial field component at $\theta=\pi / 2$ computed from (22) for several $H_{a}$. 


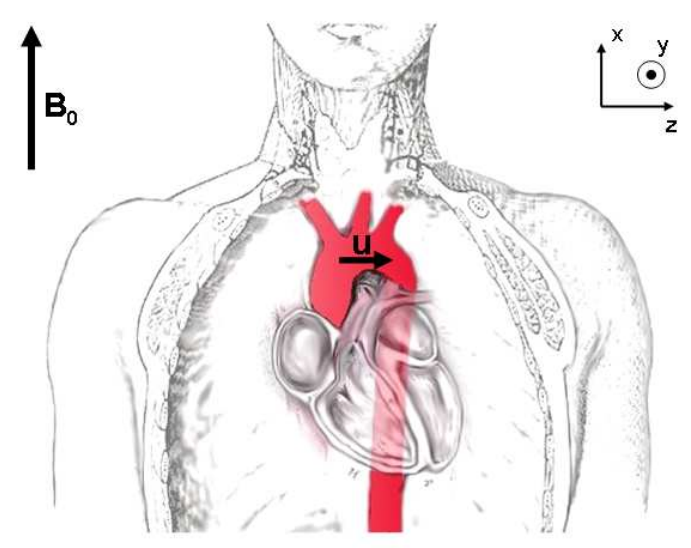

(a) Coronal plane (wikimediafoundation.org)

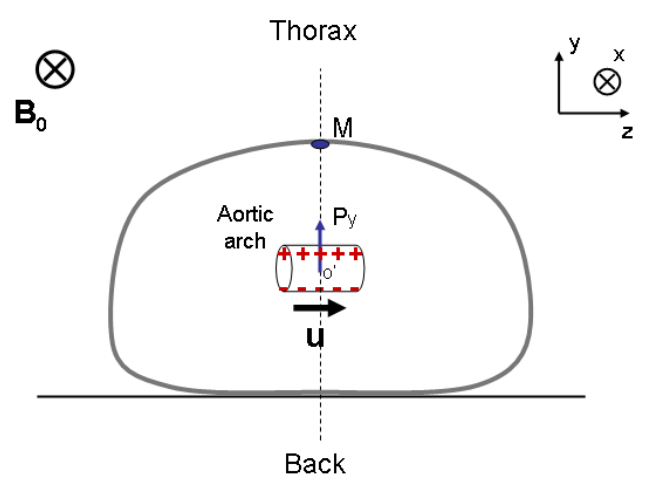

(b) Axial plane

Fig. 21. Flow and charge separation in the aortic arch. During MRI observations the static field $B_{0}$ is parallel to the body axis and the dominant magnetohydrodynamic effect is the one generated in the aortic arch 